\title{
Quantitative Mapping of Keratin Networks in 3D
}

1 Reinhard Windoffer ${ }^{1}$, Nicole Schwarz ${ }^{1}$, Sungjun Yoon $^{1}$, Teodora Piskova ${ }^{1,2}$, Michael

2 Scholkemper ${ }^{3}$, Johannes Stegmaier ${ }^{4}$, Andrea Bönsch ${ }^{5}$, Jacopo Di Russo ${ }^{1,2}$, Rudolf E. Leube ${ }^{1}$

$3 \quad{ }^{1}$ Institute of Molecular and Cellular Anatomy, RWTH Aachen University, Wendlingweg 2, 52074

4 Aachen, Germany

$5 \quad{ }^{2}$ Interdisciplinary Centre for Clinical Research, RWTH Aachen University, Pauwelsstraße 30, 52074

6 Aachen, Germany

$7 \quad{ }^{3}$ Department of Computer Science, RWTH Aachen University, Ahornstraße 55, 52074 Aachen,

8 Germany

$9{ }^{4}$ Lehrstuhl für Bildverarbeitung, RWTH Aachen University, Kopernikusstraße 16, 52074 Aachen

10 Germany

$11{ }^{5}$ Visual Computing Institute, RWTH Aachen University, Kopernikusstraße 6, 52074 Aachen

12 Germany

* Correspondence:

15 Corresponding Author

16 rleube@ukaachen.de, rwindoffer@ukaachen.de

17 Keywords: cytoskeleton, intermediate filament, keratin, retinal pigment epithelium, 18 microscopy, 3D network, image analysis, 3D visualization, virtual reality

\section{Abstract}

Mechanobiology requires precise quantitative information on processes taking place in specific 3D microenvironments. Connecting the abundance of microscopical, molecular, biochemical and cell mechanical data with defined topologies has turned out to be extremely difficult. Establishing such structural and functional 3D maps needed for biophysical modeling is a particular challenge for the cytoskeleton, which consists of long and interwoven filamentous polymers coordinating subcellular processes and interactions of cells with their environment. To date, useful tools are available for the segmentation and modeling of actin filaments and microtubules but comprehensive tools for the mapping of intermediate filament organization are still lacking. In this work, we describe a workflow to model and examine the complete 3D arrangement of the keratin intermediate filament cytoskeleton in epithelial cells both in vitro and in vivo. Numerical models are derived from super resolution 3D imaging of fluorescence-tagged keratin filaments. They are interrogated and annotated at different length scales using different modes of visualization including immersive virtual reality. In this way, information is provided on network organization at the subcellular level including mesh arrangement, density and isotropic configuration as well as details on filament morphology such as bundling, curvature and orientation. We show that the comparison of these parameters helps to identify, in quantitative terms, similarities and differences of keratin network organization in epithelial cell types defining subcellular domains, notably basal, apical, lateral and perinuclear systems. The described 
approach and the presented data are pivotal for generating mechanobiological models that can be experimentally tested.

\section{Introduction}

41 Quantification of cellular properties is a central task of cell biology (Howard, 2014; Liberali and

42 Pelkmans, 2012; Lidke, 2017; Lippincott-Schwartz, 2014; Piston, 2014; Roca-Cusachs et al., 2017).

43 It is necessary for scientific analysis, testing and model building. The structural diversity of eukaryotic cells supports their complex functions and is essential to provide local microenvironments, which facilitate and enhance the probability of highly specialized molecular interactions and reactions. The cytoskeleton is a key determinant of the necessary spatial organization. It consists of filamentous polymers, which assemble into microtubules, actin filaments and intermediate filaments. The resulting networks are interlinked and connected to intra- and extracellular targets, including cytoplasmic organelles and specialized plasma membrane sites. In this way, they act as structural and mechanical connectors between extra- and intracellular forces. The participating structures and polypeptides have been referred to as the mechanobiome (Kothari et al., 2019; Parajón et al., 2021; Wang et al., 2014), which defines the mechanical setting of a cell. The properties of several mechanobiome components have already been quantified including their turnover and mechanical features at different levels. Comprehensive quantitative information on entire cytoskeletal networks, however, is missing for most components and, consequently, only little is known about the distribution, local composition, mesh size, orientation and mechanical properties of cytoskeletal networks at the subcellular level. Therefore, there is an urgent need to describe cytoskeletal network architectures in quantitative terms at subcellular resolution. To this end, we present a novel approach to create the complete digital representation of keratin intermediate filament networks in three dimensions (3D), providing maps and quantitative measurements of the entire keratin network in cultured cells and in cells within their native tissue context.

The intermediate filament cytoskeleton consists of polypeptides, which are expressed in a tissue- and context-specific manner. The largest group of intermediate filaments, the keratin intermediate filaments are the main cytoplasmic networks in epithelial tissues. They confer, together with their associated desmosomal cell-cell and hemidesmosomal cell-extracellular matrix adhesions, mechanical resilience (Hatzfeld et al., 2017; te Molder et al., 2021). The human genome contains 54 keratin genes, which are expressed in epithelia and epidermal appendages in a cell type-specific pattern (Jacob et al., 2018). Keratin filaments are highly flexible and extensible (Block et al., 2015). They form hollow tubes with remarkable variability in local diameter and ultrastructure (Weber et al., 2021). Keratin filaments generate branched bundles of different thickness forming anisotropic networks formed (Beil et al., 2005; Martin et al., 2016). The 3D organization of keratin networks differs profoundly between different epithelia: E.g., the simple epithelial cells lining the intestine contain a well-defined lateral and apical network, keratinocytes contain a dense 3D network encompassing the entire cytoplasm, and hepatocytes have a prominent submembraneous network (Schwarz et al., 2015; Strnad et al., 2008). Network organization is furthermore affected by mechanical stress (Beriault et al., 2012; Felder et al., 2008; Fois et al., 2013; Karsch et al., 2020; Lutz et al., 2020; Quinlan et al., 2017) and differentiation-status (Coch and Leube, 2016; Iwatsuki and Suda, 2010; Leube et al., 2017; Quinlan et al., 2017; Tateishi et al., 2017). In addition, individual proteins such as Ndell and plectin have been shown to regulate keratin network organization (Kim et al., 2021; Moch et al., 2016; Osmanagic-Myers et al., 2006). Knowledge of the 3D keratin network organization in single epithelial cells is therefore important to understand and characterize the 
82 particular functional status of a given cell within its complex tissue context under different

83 conditions.

84 Segmentation of cellular elements is a key task in biological image analysis. Its application to

85 specific structures, however, can be quite difficult and requires appropriate adjustment. Networks of

86 curvilinear objects such as keratin networks are ubiquitous ranging from cytoskeletal filaments at the micro- to nanoscale to blood vessels or neuronal networks at the tissue and organ level (Özdemir and Reski, 2021). Filament detection systems have been developed for vascular (Imran et al., 2019; Moccia et al., 2018; Samuel and Veeramalai, 2020) and neuronal networks (Acciai et al., 2016; Magliaro et al., 2019; Quan et al., 2016). But these tools have not been optimized for subcellular structures and are specialized to detect branched, tree-like structures. The fishnet-like organization of keratin networks poses novel challenges, which are further confounded by the variably spaced branching points connecting filaments of different thickness and curvature.

A wide range of specialized tools are available for segmentation of cytoskeletal actin (Alioscha-Perez et al., 2016; Nowak et al., 2020), which also work in 3D (Haspinger et al., 2021). Further tools have been developed for the segmentation of microtubules (Faulkner et al., 2017) and their prokaryotic homologue FtsZ (Liu et al., 2019; Özdemir and Reski, 2021). Recently, a tool was described that is suitable for segmentation of cytoskeletal networks including keratin networks using data obtained by electron microscopy and super-resolution fluorescence microscopy (Flormann et al., 2021). This tool, however, lacks 3D capability and does not capture information on filament thickness. Another segmentation tool for filamentous networks in electronmicroscopic images was also recently reported (Dimchev et al., 2021). Until now, however, no tools are available that segment 3D fluorescent image data of keratin filament networks and transform them into a numerical graph model.

To segment keratin filament network and generate a data structure with defined nodes and internodal regions (= segments) we used the open-source program TSOAX. TSOAX has been used for 3D segmentation of actin networks using fluorescence images of yeast cells (Xu et al., 2019). It is an implementation of SOAX (Xu et al., 2015, 2011), which was developed from the Stretching Open Active Contours (SOAC) algorithm (Li et al., 2010, 2009b, 2009a). SOACs are open curves, which start at segment tips and delineate the filament centers. We now show that TSOAX can be used to

110 reliably segment keratin networks in entire cells. TSOAX also provides density information of the 111 identified keratin filaments. However, the association of nodes and segments is not detected satisfactorily as the generated TSOAX snakes are not separated consistently at filament nodes and therefore hinder statistical analysis. We therefore developed additional tools (KerNet) for optimal network representation on a node-segment structure. The combination of the established segmentation algorithms (TSOAX) and the additional KerNet analysis tools helped to create accurate numerical representations of keratin network topology.

117 The developed image analysis tools were applied to super-resolution fluorescence image stacks of 118 keratin networks in single cells either in cultured epithelial monolayers or within their native tissue context. We show that the image analysis tools provide cell type-specific information on filament mesh characteristics as well as information on filament thickness, directionality and connectivity in 3D. Cinematic rendering and immersive visualization make analysis of network heterogeneity accessible at a hitherto unknown quality and precision of 3D reconstruction. 


\subsection{Cell culture}

126 Madin-Darby Canine Kidney (MDCK) cells were stably transfected with a plasmid encoding human

127 full-length keratin 8 with an EYFP tag at its carboxyterminus (Windoffer et al., 2004; recloned into

128 pEYFP-N1 (Clontech) with BamHI and EcoRI). Resulting cell clone H9 was used for the analyses

129 described in this paper. Cells were grown in Dulbecco's Modified Eagle Medium (DMEM, Sigma-

130 Aldrich) with 10\% (v/v) fetal calf serum (Pan Biotech) including $700 \mu \mathrm{g} / \mathrm{ml}$ G418 (Sigma-Aldrich)

131 for selection. Vital MDCK cells were imaged after 3 days when the monolayer had reached complete

132 confluence.

133 Immortalized human HaCaT keratinocyte cell clone B10 expressing EYFP-tagged human keratin 5

134 (HK5-EYFP) has been described (Moch et al., 2013). The cells were grown in DMEM containing 1-

135 alanyl-glutamine (Sigma-Aldrich) supplemented with 10\% (v/v) fetal calf serum (SeraPlus; PAN

136 Biotech) and passaged as described (Moch et al., 2013). Vital HaCaT cells were imaged $24 \mathrm{~h}$ after.

\section{$137 \quad 2.2 \quad$ Preparation of retinal pigment epithelium}

138 Retinas were prepared from homozygous keratin 8-YFP knock-in mice, which synthesize

139 fluorescence-tagged keratin 8 from the modified endogenous gene locus (Schwarz et al., 2015). Mice

140 were sacrificed by cervical dislocation and eyes were enucleated using curved forceps. Eyeballs were

141 then fixed with $2 \%$ paraformaldehyde in rotation at $4{ }^{\circ} \mathrm{C}$ for $4 \mathrm{~h}$. For retina flat-mount preparation, the

142 eye balls were dissected as previously described (Claybon and Bishop, 2011). Briefly, cornea and

143 lens were removed and the eyecup was cut into a multileaved flower-shape. The neural retina was

144 removed and the remaining tissue was mounted on a microscope slide with Mowiol (Sigma-Aldrich).

\section{$145 \quad 2.3 \quad$ Image recording}

146 To obtain statistically relevant data, ten image data sets were recorded of each cell type with a Zeiss 147 LSM710 using airyscan mode (Carl Zeiss, Jena, Germany). An oil immersion Plan-Apochromat 148 63x/1.4-NA, DIC M27 objective (Carl Zeiss) was used. Fluorescence was detected with an $488 \mathrm{~nm}$ 149 argon laser (module LGK 7872 ML8) and filter set BP 495-550 using 2.5\% power. Optimal factory 150 settings for $\mathrm{z}$-stacks were used resulting in a voxel size of $\mathrm{xy}=0.044$ to $0.065 \mu \mathrm{m}$ and $\mathrm{z}=0.17$ to 0.2 $\mu \mathrm{m}$. No further image optimization was used. To assure that only single cells were analyzed, the fluorescence in individual cells was cropped from confluent MDCK monolayers and retinal pigment epithelium (RPE) using Fiji standard tools. Cell borders were manually defined for each z-slice and

154 the cell exterior was set to a grey value of 0. Wild-type HaCaT and HK5-EYFP producing HaCaT 155 clone B10 cells were mixed prior to prevent overlap of fluorescence signals in neighbouring cells.

\subsection{Segmentation of image stacks}

157 TSOAX software (Xu et al., 2019, 2015, 2011) was used for the segmentation of filaments from the

158 recorded 3D image stacks (Suppl. Figure 1A). TSOAX facilitates quantitative analysis of network

159 dynamics of multi-dimensional biopolymer networks imaged by various microscopic imaging

160 modalities. The underlying methods of TSOAX include multiple Stretching Open Active Contour

161 (SOAC) models for extraction and a combined local and global graph-matching framework (Li et al.,

162

163

164 2010, 2009b, 2009a). For optimal results, the default TSOAX parameters were used, with the exception of 'external-factor' which was set to 2 based on internal testing. Since computation time can be extremely long, TSOAX was executed in parallel instances on the supercomputer CLAIX at RWTH Compute Cluster (https://www.itc.rwth-aachen.de/go/id/eucm). 


\subsection{Calculation of a segment/node-based network model}

To get a perfect network representation it is necessary to divide the network into defined pieces (= segments) that do not cross each other, and can be connected at their ends by nodes. Segmentations using TSOAX defines polygonal chains with $n$ vertices (=snakes). Each vertex is defined in $\mathrm{x}, \mathrm{y}, \mathrm{z}$ and has an associated density (= fluorescence intensity). However, the snakes are not associated with each other via nodes and may cross each other. (Suppl. Figure 1B). To obtain a comprehensive and coherent numerical network definition from snakes, KerNet software was developed. It generates a graph representation consisting of segments ( edges) and nodes. To compute such a connected network representation, vertices of a snake that lie within $\varepsilon=1.1 \mathrm{x}$ voxelsize distance of one another are combined to form a node. If more than two vertices are within $\varepsilon$ distance, a candidate set is created. Any vertex, which is at most $\varepsilon$ away from a vertex in the candidate set, is added to the set until this is no longer possible. Then all vertices in the candidate set are combined to form a single node. Finally, any start or end vertex of a segment is a node. If two nodes are connected by a part of a snake then a segment connects the two nodes in the network. In contrast to classical edges, which are defined as lines connecting the nodes, each segment is a list of vertices (x,y,z) and their associated densities. The segments therefore inform about the trajectory of the snake between its two endpoints and the density progression along the segment. To summarize, nodes represent the first and last vertex of a segment and mark the connection between segments whose endpoints are close (Suppl.

Figure 1C). All segments and nodes together represent a Euclidean 3D network map of a given cell.

\section{$186 \quad 2.6 \quad$ Validation of segmentation}

187 For validation of the segmentation, the original fluorescence image stacks were compared with the 188 segmented 3D data. All vertices retrieved from KerNet were plotted into the original image stack. The z-values of the vertices were rounded to the nearest $\mathrm{z}$-slice and all segment vertices were drawn as discs with a diameter proportional to their brightness. To simulate the blurriness of the raw images, the vertex stacks were 3D-Gauss filtered using Fiji (values 5,5,3).

193 For inspection of the 3D network representation, the xyz-position data of segments were exported 194 into OBJ files (http://www.martinreddy.net/gfx/3d/OBJ.spec), which, in turn, can be imported into 195 3D rendering programs. For the representation of segment brightness as renderable tubes, density 196 properties of segments were compiled into 3ds Max (Autodesk) script files that translate segment 197 brightness into tube thickness, resulting in renderable splines for each segment. In some instances, 198 random colors were assigned to each segment (Suppl. Figure 1D). Finally, 3ds Max rendering was 199 used for the generation of stills and videos.

\section{$200 \quad 2.8$ Virtual reality}

201 For further visual inspection of the keratin networks, a basic Virtual Reality (VR) application was 202 designed. The VR application was implemented using Unreal Engine 4 (https://www.unrealengine.com/en-US/). It displays the datasets of cells as 3D models. The individual filaments are visualized as tubes. Their brightness is encoded in the tubes' thickness. The resulting structure of interconnected tubes can then be visually inspected in VR, allowing a detailed view on the digitalized subcellular network in any magnification. Individual filaments can be 
interactively assigned to one of several distinct classes, pre-defined by the domain expert. Color-

coding is then used to visually differentiate the classes in the immersive visualization.

209 During our research, we used the HTC Vive Pro headset to manually classify the filaments of a cell.

210 Afterwards, the aixCAVE at RWTH Aachen University was used to collaboratively discuss and

211 adjust the classification.

\section{$212 \quad 2.9 \quad$ Numerical analysis and visualization}

213 Matlab routines were used for further analysis of network properties. Up to 150000 vertices may be present per cell. Segment length, distance between nodes, bending, curvature and apparent persistence length of segments were calculated. Bending was defined as the ratio beween the shortest distance between the segment endpoints and the actual segment length. The curvature was calculated as the 2nd derivative of the smoothed tangent vector with respect to the arc length (Sternberg, 2012). Since persistence length can only be calculated in free moving segments and the segments are restricted in their movement by the nodes in our situation, we calculated an apparent persistence length as described (Doi and Edwards, 1990).

Statistical analyses were performed with Matlab and GraphPad Prism software. For non-Gaussian distribution, Mann-Whitney test was used. Values are expressed as the mean \pm SD. * represents a pvalue of $\mathrm{p}<0.05, * * \mathrm{p}<0.01, * * * \mathrm{p}<0.001$ and $* * * * \mathrm{p}<0.0001$. The differences were considered significant when $p<0.05$. Non-significant differences were indicated by n.s.

To visualize segment properties such as length or curvature, the respective values were plotted and color-coded at the vertex location resulting in heat maps showing the distribution of the selected property. Here, Fiji scripts were used to plot color-coded properties into xyz coordinates of vertices.

For calculation of cell size, a threshold was used, that separated the background fluorescence inside and outside of cells.

\subsection{Directional vectors}

232 The directionality of segments was calculated for the keratin network of single cells in two ways:

(i) Since the start and end points of segments are randomly orientated, segments with an orientation difference of 180 degrees were equally orientated. Therefore, normalized histograms were plotted for azimuth and elevation from 0-180 degrees. Next, we calculated the deviation of the histogram from a histogram with an even distribution of orientations where each degree has the same frequency. Finally, the difference between the segment histogram and the even distributed histogram was calculated as the sum of the differences in each bin.

(ii) Keeping the segments in their original orientation we analyzed the position of segments in segments in xyz. It was then determined whether the start point or end point of each segment was closer to the cell center. The segment was then moved to the cell center such that the closer point was placed to the cell center. In this way, all segments originate at the cell center and direct outwards. The resulting radial histograms were plotted to depict the distribution of segments in xy and yz directions. 
245 Treating each segment as a vector extending from the cell center to the segment's end, the sum vector

246 of all segments was calculated and plotted.

\section{$247 \quad 2.11$ Software, original and processed data}

248 Software, original and processed data are available at http://kernet.rwth-aachen.de/ and

249 https://github.com/VRGroupRWTH/Zytoskelett.

\section{RESULTS}

\section{$252 \quad 3.1 \quad$ Recording of the keratin intermediate filament network in 3D}

253 In a first step, we wanted to generate high-resolution fluorescence data that would be suitable for 254 image analysis of single filaments and filament bundles and would represent keratin filament networks in different epithelial cell types. We selected three non-carcinogenic epithelial cell types: $(i)$ Canine kidney epithelial MDCK cells producing YFP-tagged keratin 8 (subclone H9) were chosen as representatives of polarized simple epithelial cells. MDCK cells form a one-layered coherent epithelium with the cuboidal to cylindrical cells attached to each other by highly-organized junctional complexes. Intracellularly, the keratin filaments have a complex arrangement consisting of a dense subapical network, perinuclear and radial keratin filaments and interdesmosomal filaments below the plasma membrane (Quinlan et al., 2017). (ii) Spontaneously immortalized human epidermal keratinocytes expressing YFP-tagged keratin 5 (HaCaT B9) were chosen as representatives of squamous epithelial cells-(Boukamp et al., 1988) (Moch et al., 2013). They contain pancytoplasmic keratin filament networks whose 3D organization is only partially elucidated (Moch et al., 2020). (iii) To examine the 3D organization of the keratin intermediate filament network in its native tissue context, we made use of the recently described knock-in mouse line expressing YFP-tagged keratin 8. Retinal epithelium cells (RPE) were selected for the analysis because of their well-delineated cytoplasmic network (see below). High resolution airyscan confocal image stacks were prepared from each cell type.

\subsection{Transformation of 3D fluorescence recordings of keratin filaments into digital network models}

The recorded image stacks were transformed into Euclidean 3D maps using TSOAX and the KerNet nodes describe the entire keratin networks of single cells. The segments represent keratin filament bundles of different thickness and length. These segments are defined as 3D polygonal chains of vertices, each of which has a specific xyz-position and brightness. The first and last vertices of a segment are defined as nodes. Nodes are either shared by adjacent connecting segments within a filament or are positioned at the end of a filament in case of loose ends. Note that keratin filaments consist of multiple consecutive segments. To validate the quality of the numerical transformation, all vertices were plotted onto the maximum intensity projection of the fluorescence image stack. Figure 1A shows the maximum intensity projection of the fluorescence in an MDCK cell as an example highlighting selected regions of interest at higher magnification. Separating the regions above and below the nucleus (apical and basal, respectively) furthermore allowed to distinguish the different keratin network structures in these subcellular domains. Visual inspection showed a high degree of overlap between the recorded fluorescence and the derived numerical positions of vertices (dotted lines) at all subcellular locations. 
To obtain more detailed comparisons of the calculated vertices with the fluorescence data, vertices were first plotted in 3D and their brightness was encoded as filament diameter. The 3D plots were then convolved by Gaussian blurring to simulate the microscope's point spread function. Figure 1B shows the very good match of the convolved vertices in terms of position and brightness in comparison to the maximum intensity projection of the fluorescence image. To assess the overall reliability of the digital transformation, the validation steps were applied to all cells selected for the study (Suppl. Figure 2). Additional slice-by-slice comparisons are presented exemplarily in Video 1 further demonstrating the good correlation between the recorded and derived numerical representation of the keratin filament network in 3D.

For close inspection of the keratin filament network in its subcellular complexity, we used cinematic rendering. The rendering displays the network as a true 3D model including the brightness of filaments which are shown as tubes with different degrees of thickness thus providing information on the segments in a 3D-viewer. To distinguish individual segments, each segment was randomly colorcoded (Figure 1C).

\subsection{Comparison of 3D cinematic keratin filament renderings in MDCK, HaCaT and RPE cells}

Renderings of keratin filament networks in MDCK, HaCaT and RPE cells are exemplarily shown from different views in Figure 2 and Videos 2-4. Interactive 3D renderings of all cells are available at kernet.rwth-aachen.de. Furthermore, immersive visualization was used to provide unrivaled impressions of the spatial arrangements of the keratin network in stereoscopic 3D (Figure 3). The multidimensional visualizations revealed hallmark features of the different keratin filament networks.

Thus, an apical and a basal keratin filament network domain can be distinguished in MDCK cells (Video 2). The major difference between both domains are the thicker keratin bundles in the basal domain. Each network is characterized by a regular mesh pattern. The lateral keratin filament network is more heterogenous and dominated by filaments running between apical and basal. Circumferential subcortical keratin filaments are mainly restricted to the apical and basal network domains. In the cell interior, keratin filaments form a network around the nucleus. It is best developed on the basal side with highly bundled filaments, many of which are rather straight.

316 HaCaT cells are extremly flat and do not show a clearly seperated apical and basal networks and lack 317 a distinct lateral network (Video 3). A prominent feature of their keratin filament network are long 318 filament bundles that often run in parallel. They are absent above and below the nucleus but enclose 319 the nucleus laterally.

320 The keratin network is less dense in RPE cells than in the other two cell types (Video 3). It is prominent in the cytoplasmatic apical domain, which faces the photoreceptor cells in the retina. It is also very well developed around the nucleus. In contrast to MDCK cells, the perinuclear network contains thicker filaments at the apical than the basal side. The basal cytoplasmatic domain is mostly devoid of keratin filaments. Some filaments run through the cytoplasm from basal to apical.

In conclusion, rendering of the keratin filament networks in three cell types revealed very different features. Especially with the help of VR, classification of network domains becomes feasable. 


\subsection{Comparison of keratin filament brightness distribution patterns}

330

331

332

333

334

335

336

337

338

339

340

341

342

343

344

345

346

347

348

349

350

351

352

353

354

355

356

357

358

359

360

361

362

363

364

365

366

367

368

369

370

371

The numerical network representations were used to quantify keratin filament properties. A prominent property, which was already graphically encoded in the 3D-models, is filament brightness as a measure of filament thickness. The assumption is that filament thickness is a reliable indicator of filament bundling which can now be easily quantified throughout entire cells. The histograms in Figure 4A-C depict the spread of filament segment brightness in MDCK, HaCaT and RPE cells. The quantifications show that filament segments with a medium brightness dominated in MDCK cells with a near Gaussian distribution, whereas thin filaments were prevalent in HaCaT and RPE cells. Thin filaments were most abundant in $\mathrm{HaCaT}$ cells.

Maps of filament brightness are shown in Figure 4D-F for selected examples of each cell type at low and high magnification. In each case, thin filaments were numerous in the cell periphery. This may be due to the increased de novo single filament formation in these regions (Windoffer et al., 2004, 2006, Moch et al., 2013). Some areas, such as those shown at the right margin of the MDCK cell in Figure 4D, however, presented rather thick keratin filament bundles, which may represent matured interdesmosomal keratin filaments (Quinlan et al., 2017). The most heterogeneous network in terms of filament thickness was encountered in MDCK cells. The animation in Video 2 clearly shows that filaments in the basal network of the cell are thicker than that in the apical network. In HaCaT cells, enrichment of thin filaments was visible around the nucleus (Figure 4E) whereas thicker filaments were detectable in the cytoplasm, many of which appeared to be arranged in parallel (see also Video 4). In contrast, thick filament bundles surrounded the nucleus of RPE cells (Figure 4F; Video 5). Furthermore, thick bundles were more frequent above the nucleus, i.e., at the part of the cell facing the photoreceptors. Together, the analyses of the digitalized networks revealed major differences in the extent and distribution of keratin filament bundling suggesting that the keratin network is organized to withstand and cope with different types of mechanical stress.

\subsection{Number and length of keratin filament segments and overall keratin filament length in single cells}

The number and length of individual keratin filament segments are key measurements of network organization. Significantly more segments were detected in $\mathrm{HaCaT}$ cells than in the other cell types (Figure 5A). The average segment length was shortest in MDCK cells $(0.80 \pm 0.04 \mu \mathrm{m})$, longest in RPE cells $(0.88 \pm 0.05 \mu \mathrm{m})$ and intermediate in HaCaT cells $(0.83 \pm 0.13 \mu \mathrm{m})$ (Figure 5B).The combined length of all segments per cell (Figure 5C) was similar in MDCK $(1.63 \pm 0.41 \mathrm{~mm})$ and RPE cells $(1.57 \pm 0.48 \mathrm{~mm})$ but was more than twice longer in HaCaT cells $(4.40 \pm 1.53 \mathrm{~mm})$. To see whether this difference was due to different cell dimensions, the cell volumes were determined for all three cell types. Significant differences could be detected, i.e., $4.65 \pm 1.13 \mu \mathrm{m}^{3}$ for MDCK, $9.16 \pm 6.10$ $\mu \mathrm{m}^{3}$ for $\mathrm{HaCaT}$ and $2.73 \pm 0.95 \mu \mathrm{m}^{3}$ for RPE (Suppl. Figure 3A). Taking the different cell volumes into account, filament density, i.e. filament length per volume, was comparable in $\mathrm{HaCaT}$ $\left(604.82 \pm 304.20 \mu \mathrm{m} / \mu \mathrm{m}^{3}\right)$ and RPE cells $\left(585.60 \pm 63.97 \mu \mathrm{m} / \mu \mathrm{m}^{3}\right)$, while it was considerably lower in MDCK cells (355.24 $\left.\pm 56.49 \mu \mathrm{m} / \mu \mathrm{m}^{3}\right)$ (Figure 5D). Given that the human body has $\sim 0.14 \times 10^{12}$ epidermal cells (Bianconi et al., 2013), the entire length of epidermal keratin filament bundles in an individual should be $\sim 600000 \mathrm{~km}$. Assuming a bundling rate of 19 filaments per filament bundle (Hémonnot et al., 2016) there are $\sim 1.14 \times 10^{7} \mathrm{~km}$ of single filaments keratin filaments in the epidermis of a human body. 


\subsection{Branching and intersection of keratin filaments}

374

375

376

377

378

379

380

381

382

383

384

385

386

387

388

389

390

391

392

393

394

395

396

397

398

Next, we analyzed branching and intersection of keratin filament bundles. -Different types of branching could be envisioned depending on the number of segments per node. Three segments per node would define a Y-shaped branch, whereas four segments would define an X-shaped intersection of two filaments (Suppl. Figure 1C). More than four branches would result in a star-like configuration. Our analyses show that the Y-shaped organization (three segments per node) was by far the most frequent configuration $(\sim 60 \%)$ followed by the $\mathrm{X}$-shaped (four segment per node) arrangement $(\sim 30 \%)$ and rarely higher degrees of branching $(\sim 10 \%)$ (Figure 6).

\subsection{Bending of keratin filaments}

Bending of keratin intermediate filaments is of interest as it may provide information on material properties of filaments and may indicate whether filaments are under longitudinal compression or tension. First, we calculated the distance/length ratio of segments (Figure 7A). The highest ratios were observed in MDCK $(0.92 \pm 0.09)$ and HaCaT $(0.92 \pm 0.08)$, which were significantly higher than in RPE cells $(0.90 \pm 0.10)$ indicating that the filaments in RPE cells are less straight. This interpretation was confirmed by curvature determination of segments (Figure 7B). The mean curvature of segments was significantly higher in RPE cells $\left(3.30 \pm 0.15 \mu^{-1}\right)$ than in MDCK cells $\left(2.54 \pm 0.11 \mu \mathrm{m}^{-1}\right)$ and $\mathrm{HaCaT}$ cells $\left(2.97 \pm 0.72 \mu \mathrm{m}^{-1}\right)$. The curvature distribution in HaCaT cells was more widely spread than in the other two cell types. Maps of curvature distribution are shown from two different perspectives in Figure 7C-E revealing complex patterns. Finally, we determined the apparent persistence length of keratin filament segments being aware that the resulting values are difficult to interpret, since segments are not free to move but are connected to other segments within the network. Suppl. Figure 3B shows that the calculated values $(\sim 2.6 \mu \mathrm{m})$ do not differ significantly between the three cell types. As expected, they are higher than the experimentally determined persistence length of single filaments (cf. (Block et al., 2015)).

\subsection{Keratin filament segment orientation}

To calculate how evenly the segments are orientated in space, the azimuth (= horizontal orientation) and the elevation (= vertical orientation) of segments were calculated for each cell. The histograms in Figure 8A-F show examples of the azimuth and elevation distribution in single MDCK, HaCaT and RPE cells. Comparing the determined values with the expected values for segments orientated evenly in all directions, shows that the azimuth distribution is quite even in MDCK and RPE cells but not in HaCat cells. All cells have an uneven distribution of vertical orientation, indicating that segments are preferentially oriented horizontally. Compilations of the analyses for all cells (Figure 8G, $\mathbf{H}$ ) confirmed the single cell data and affirmed that the azimuth distribution in HaCaT is more oriented than in MDCK or RPE cells. Furthermore, the elevation orientation is more directed in MDCK and $\mathrm{HaCaT}$ cells than in RPE cells. Mapping the horizontal segment orientation in selected cells (Figure 8I-L) reveals a random pattern in MDCK and RPE and highlights parallel-arranged filaments in HaCaT. 
412 To obtain insight about the relationship between segment directionality and cell shape, the orientation

413 of the segments was analyzed with respect to the cell center. To this end, all segments were

414 translocated to the cell center and their spatial distribution was plotted in radial 2D histograms. As

415 expected, MDCK and RPE cells showed a homogenous distribution of horizontal segment orientation

416 in the $\mathrm{x} / \mathrm{y}$ plane while $\mathrm{HaCaT}$ cells were more polarized (Figure 9A-C). In the vertical direction (y/z

417 plane) horizontally oriented segments predominate in MDCK and HaCaT cells while segments are

418 more uniformly oriented in RPE cells (Figure 9D-F).

419 To compare the 3D-isotropy of entire keratin filament networks a sum vector of all segments was

420 constructed for each cell. To this end, the orientation vectors of segments were translocated to the

421 cell center as described above and mapped with the length of each segment. Figure 9G-I shows the

422

423

424

425

426

resulting sum vectors in each plane together with corresponding views of the segmented keratin filament networks. The sum vectors in MDCK and RPE cells are relatively small, indicating an isotropic orientation of the respective keratin networks. In contrast, the sum vectors in HaCaT cells are significantly much longer, indicating a higher degree of anisotropy of the keratin network (Figure 9K).

\section{DISCUSSION}

With this work we provide, for the first time, experimentally-derived numerical 3D representations of entire keratin networks in eukaryotic cells. 3D fluorescence data were used to define segments that are connected by nodes. This enabled us to compare local properties of keratin intermediate filament networks such as filament thickness, curvature and orientation in different cell types in vitro and in vivo. It further facilitated comprehensive comparisons of entire networks by quantifying parameters such as entire filament length, degree of filament connectivity and spatial network orientation.

A critical aspect of the work was evaluation of the quantification process. Since synthetic 3D data of cellular keratin networks were lacking, the quality of segmentation was evaluated by visual inspection of superimposed imaging and segmentation data demonstrating that the location and intensity of filaments were mapped with high precision and reliability. The subsequent allocation of nodes and segments was visually assessed in rendered 3D models (cinematic rendering) again revealing very good agreement of the assignments. Nevertheless, the numerical representation has its limitations in accuracy. Proper segment and node detection was particularly challenging in $\mathrm{HaCaT}$ cells because of their complex network geometry in combination with low filament bundling. Extreme bending at the single filament level with bending radii of $100 \mathrm{~nm}$ (Weber et al., 2021) are not detected in the present set-up due to the resolution limit of the airyscan. Mapping of keratin filaments at the cell edges was also difficult in some regions, mostly because of the manual assignment of cell borders in the confluent cell layers with tightly attached and partially overlapping neighboring cells. In cultured cells, mixing of fluorescent and non-fluorescent cells could overcome this problem. Segmentation quality may be further improved in the future by using other and more powerful super-resolution techniques than the airyscan technology used in the present work.

A major advance of the current report is the fabrication of numerical models of the keratin filament network in 3D. The rendered 3D models can be thoroughly inspected with the help of 3D display devices such as 3D screens, virtual reality-headsets (HMDs) or Cave Automatic Virtual Environments (CAVEs). The benefits of such immersive environments have been demonstrated (Cruz-Neira et al., 1993; Laha et al., 2013). Three factors thereby improve the domain scientists' spatial judgment and thus the task performance during the visual analysis: (i) an enlarged field of 455 regard, compared to standard desktop systems, reduces clutter during the data exploration, (ii) 
stereoscopy provides supporting depth cues, and (iii) head tracking provides enhanced motion parallax.

The derived digital network models furthermore allow comparisons between cells with similar or dissimilar differentiation status (Figure 10). As a proof of concept, we paradigmatically investigated three different epithelial cell types finding significant differences in filament bundling, segment length, filament density, filament curvature and filament orientation. These parameters are a crucial and first step to build mechanical models of keratin filament networks in different cell types and functional contexts. The following list shows potential implications of the extracted features for our understanding of keratin filament network properties and functions:

- Brightness of keratin filament segments: Brightness levels correspond to filaments thickness and is therefore a measure of keratin filament bundling. The observed variation in filament bundling in the different cell types may therefore be a consequence of differences in the local force balance. This force balance is the result of intrinsic cell type-specific properties such as cell shape and arrangement of the actomyosin system and is impacted by forces imposed by neighboring cells and the extracellular matrix. This is supported by the observed differences in bundling of the apical, basal and perinuclear keratin filament networks. However, the variation in filament thickness may also reflect other properties such as the propensity of the network to reorganize, e.g., at different times of the cell cycle. Correlating the derived image data with information on mechanical keratin filament properties obtained by laser cutting experiments, force-sensor measurements or traction force analyses may help to better understand the relationship between filament bundling and mechanics.

- Length of keratin filament segments: Keratin filament segment length is an indirect measure of mesh size and may therefore describe another crucial mechanical property of the network. We were surprised to find that the segment length varied only by $10 \%$ between the different cell types and thus showed less variation than filament brightness or curvature. This means that the mesh size is fixed within a relatively small range, although the number of meshes per volume differed significantly between the different cell types. A conclusion of this observation is that mesh size may be determined by intrinsic keratin filament properties such as elastic modulus or branching capacity. However time-lapse fluorescence analyses (e.g., (Wöll et al., 2005)) have shown that small meshes can merge and enlarge by the combination of nodes from two segments, forming thicker filament bundles. Conversely, large meshes are subdivided into small meshes by recruitment of segments into a node. A self-regulatory mechanism may thus control the balance between enlargement and reduction of individual meshes.

- Branching of keratin filament segments: Experimental and mechanistic evidence has been provided for keratin filament branching (Beil et al., 2005; Feng and Coulombe, 2015; Lee et al., 2012; Martin et al., 2016; Nafeey et al., 2016). The fact that only two types of branching/intersections were detected in this study, points to a limited repertoire of connectivity. Furthermore, the Y- and Xshaped configurations may be fundamentally different. The Y-shaped joining of three segments may be linked to the recently proposed bundling mechanism involving electrostatic and hydrophobic interactions (Haimov et al., 2020). On the other hand, the X-shaped intersections may be related to the disulfide bond mediated interactions reported for keratins 5/14 (Lee et al., 2012). In this instance, preventing these links resulted in altered keratin filament network dynamics and epidermal tissue homeostasis (Feng and Coulombe, 2015; Guo et al., 2020). 
499

500

501

502

503

504

505

506

507

508

509

510

511

512

513

514

515

516

517

518

519

520

521

522

523

524

525

526

527

528

529

530

531

532

533

534

535

536

537

538

539

540

541

- Curvature of keratin filament segments: Bending of keratin filaments provides important information on whether keratins are stretched, relaxed or compressed. Keratin intermediate filaments are highly elastic (Kreplak et al., 2005; Wagner et al., 2007; Yamada et al., 2003). They can be extended under tension forming straight filaments and buckle under compression (= negative tension) (Fudge et al., 2008). The measurements of segment curvature can therefore be taken as indicators of local keratin filament tension. Straight segments were almost completely absent in all cells suggesting overall negative tension of the keratin networks. The degree of filament bending, however, differed between the different cell types indicating cell type-specific differences in intrinsic force balance and may suggest that the different networks retain different degrees of flexibility before they become stretched to function as mechanical counterbalances to strain. Curvature of keratin filaments also allowed to estimate the persistence length of network-embedded keratin filaments, i.e., the minimum length between two points on the polymer where they become independent of one another. The value of $\sim 2 \mu \mathrm{m}$ was only slightly higher than what was reported for other intermediate filaments including keratin (Block et al., 2015; Wagner et al., 2007). This is low in comparison to actin filaments $(\sim 10 \mu \mathrm{m})$ (De La Cruz and Gardel, 2015; Isambert et al., 1995; Takatsuki et al., 2014) and microtubules $(\sim 5000 \mu \mathrm{m})$ (Hawkins et al., 2010). This result emphasizes the maintained high flexibility of keratin filaments despite their connectivity through nodes.

- Orientation of keratin filaments: To understand the response of the keratin network to internal and external forces, it is important to have measures of filament orientation. We suggest that uniformly radial orientation of keratin filament segments indicates that cells experience mechanical forces that are homogenous in all directions. In contrast, a preferential orientation in one direction indicates that keratin filaments align in response to mechanical cues that may be induced by unevenly distributed cell-cell contacts, polarized cell-extracellular matrix adhesions or contractile activity. Our data therefore indicate that $\mathrm{HaCaT}$ cells are subjected to mechanical strain (uneven azimuth distribution and large sum vector), whereas MDCK and RPE are subjected to little mechanical (uniform azimuth distribution and small sum vector). Fine mapping of the distribution patterns of hemidesmosomes and desmosomes may help to understand the differences and to initiate mechanical modelling, improving previous models of keratin network, morphogenesis, dynamics and mechanics (Beil et al., 2009; Dallon et al., 2019; Latorre et al., 2018; Portet, 2013; Portet et al., 2019, 2015).

The observed high degree of segment property variability must be taken as an indication of the amazing plasticity of the keratin filament system.-The node/segment arrangement provides a unique 3D structure that can be modified in multiple ways affecting bundling, flexibility and local cross-talk. Keratin isotypes, posttranslational modifications, external and internal forces, and interactions with other cytoskeletal and cellular components may determine these properties and thereby act as modulators of network structure at different length and time scales.

\section{$5 \quad$ Citations}

Acciai L, Soda P, Iannello G. 2016. Automated Neuron Tracing Methods: An Updated Account. Neuroinform 14:353-367. doi:10.1007/s12021-016-9310-0

Alioscha-Perez M, Benadiba C, Goossens K, Kasas S, Dietler G, Willaert R, Sahli H. 2016. A Robust Actin Filaments Image Analysis Framework. PLOS Computational Biology 12:e1005063. doi:10.1371/journal.pcbi.1005063 
542

543

544

545

546

547

548

549

550

551

552

553

554

555

556

557

558

559

560

561

562

563

564

565

566

567

568

569

570

571

572

573

574

575

576

577

578

579

580

581

582

583

584
Beil M, Braxmeier H, Fleischer F, Schmidt V, Walther P. 2005. Quantitative analysis of keratin filament networks in scanning electron microscopy images of cancer cells. Journal of Microscopy 220:84-95. doi:10.1111/j.1365-2818.2005.01505.x

Beil M, Lück S, Fleischer F, Portet S, Arendt W, Schmidt V. 2009. Simulating the formation of keratin filament networks by a piecewise-deterministic Markov process. Journal of Theoretical Biology 256:518-532. doi:10.1016/j.jtbi.2008.09.044

Beriault DR, Haddad O, McCuaig JV, Robinson ZJ, Russell D, Lane EB, Fudge DS. 2012. The Mechanical Behavior of Mutant K14-R125P Keratin Bundles and Networks in NEB-1 Keratinocytes. PLoS One 7:e31320. doi:10.1371/journal.pone.0031320

Bianconi E, Piovesan A, Facchin F, Beraudi A, Casadei R, Frabetti F, Vitale L, Pelleri MC, Tassani S, Piva F, Perez-Amodio S, Strippoli P, Canaider S. 2013. An estimation of the number of cells in the human body. Annals of Human Biology 40:463-471. doi: $10.3109 / 03014460.2013 .807878$

Block J, Schroeder V, Pawelzyk P, Willenbacher N, Köster S. 2015. Physical properties of cytoplasmic intermediate filaments. Biochim Biophys Acta 1853:3053-3064. doi:10.1016/j.bbamcr.2015.05.009

Boukamp P, Petrussevska RT, Breitkreutz D, Hornung J, Markham A, Fusenig NE. 1988. Normal keratinization in a spontaneously immortalized aneuploid human keratinocyte cell line. Journal of Cell Biology 106:761-771. doi:10.1083/jcb.106.3.761

Claybon A, Bishop AJR. 2011. Dissection of a Mouse Eye for a Whole Mount of the Retinal Pigment Epithelium. JoVE (Journal of Visualized Experiments) e2563. doi:10.3791/2563

Coch RA, Leube RE. 2016. Intermediate Filaments and Polarization in the Intestinal Epithelium. Cells 5:32. doi:10.3390/cells5030032

Cruz-Neira C, Sandin DJ, DeFanti TA. 1993. Surround-Screen Projection-Based Virtual Reality: The Design and Implementation of the CAVEIn_Proc. 20th Annu. Conf. Comput. Graph. Interactive Techn. pp. 135-142.

Dallon JC, Leduc C, Etienne-Manneville S, Portet S. 2019. Stochastic modeling reveals how motor protein and filament properties affect intermediate filament transport. Journal of Theoretical Biology 464:132-148. doi:10.1016/j.jtbi.2018.12.022

De La Cruz EM, Gardel ML. 2015. Actin Mechanics and Fragmentation. J Biol Chem 290:1713717144. doi:10.1074/jbc.R115.636472

Dimchev G, Amiri B, Fäßler F, Falcke M, Schur FK. 2021. Computational toolbox for ultrastructural quantitative analysis of filament networks in cryo-ET data. bioRxiv 2021.05.25.445599. doi:10.1101/2021.05.25.445599

Doi M, Edwards SF. 1990. The Theory of Polymer Dynamics, International Series of Monographs on Physics. Oxford Science Publications.

Faulkner C, Zhou J, Evrard A, Bourdais G, MacLean D, Häweker H, Eckes P, Robatzek S. 2017. An automated quantitative image analysis tool for the identification of microtubule patterns in plants. Traffic 18:683-693. doi:10.1111/tra.12505

Felder E, Siebenbrunner M, Busch T, Fois G, Miklavc P, Walther P, Dietl P. 2008. Mechanical strain of alveolar type II cells in culture: changes in the transcellular cytokeratin network and adaptations. Am J Physiol Lung Cell Mol Physiol 295:L849-L857.

doi:10.1152/ajplung.00503.2007 
585

586

587

588

589

590

591

592

593

594

595

596

597

598

599

600

601

602

603

604

605

606

607

608

609

610

611

612

613

614

615

616

617

618

619

620

621

622

623

624

625
Feng X, Coulombe PA. 2015. A role for disulfide bonding in keratin intermediate filament organization and dynamics in skin keratinocytes. J Cell Biol 209:59-72. doi: $10.1083 /$ jcb.201408079

Flormann DAD, Schu M, Terriac E, Thalla D, Kainka L, Koch M, Gad AKB, Lautenschläger F. 2021. A novel universal algorithm for filament network tracing and cytoskeleton analysis. FASEB J 35:e21582. doi:10.1096/fj.202100048R

Fois G, Weimer M, Busch T, Felder ET, Oswald F, Wichert G von, Seufferlein T, Dietl P, Felder E. 2013. Effects of keratin phosphorylation on the mechanical properties of keratin filaments in living cells. The FASEB Journal 27:1322-1329. doi:10.1096/fj.12-215632

Fudge D, Russell D, Beriault D, Moore W, Lane EB, Vogl AW. 2008. The Intermediate Filament Network in Cultured Human Keratinocytes Is Remarkably Extensible and Resilient. PLOS ONE 3:e2327. doi:10.1371/journal.pone.0002327

Guo Y, Redmond CJ, Leacock KA, Brovkina MV, Ji S, Jaskula-Ranga V, Coulombe PA. 2020. Keratin 14-dependent disulfides regulate epidermal homeostasis and barrier function via 143-3 $\sigma$ and YAP1. eLife 9:e53165. doi:10.7554/eLife.53165

Haspinger DC, Klinge S, Holzapfel GA. 2021. Numerical analysis of the impact of cytoskeletal actin filament density alterations onto the diffusive vesicle-mediated cell transport. PLOS Computational Biology 17:e1008784. doi:10.1371/journal.pcbi.1008784

Hatzfeld M, Keil R, Magin TM. 2017. Desmosomes and Intermediate Filaments: Their Consequences for Tissue Mechanics. Cold Spring Harb Perspect Biol 9:a029157. doi:10.1101/cshperspect.a029157

Hawkins T, Mirigian M, Selcuk Yasar M, Ross JL. 2010. Mechanics of microtubules. Journal of Biomechanics, Special Issue on Cell Mechanobiology 43:23-30. doi:10.1016/j.jbiomech.2009.09.005

Hémonnot CYJ, Reinhardt J, Saldanha O, Patommel J, Graceffa R, Weinhausen B, Burghammer M, Schroer CG, Köster S. 2016. X-rays Reveal the Internal Structure of Keratin Bundles in Whole Cells. ACS Nano 10:3553-3561. doi:10.1021/acsnano.5b07871

Howard J. 2014. Quantitative cell biology: the essential role of theory. MBoC 25:3438-3440. doi:10.1091/mbc.e14-02-0715

Imran A, Li J, Pei Y, Yang J-J, Wang Q. 2019. Comparative Analysis of Vessel Segmentation Techniques in Retinal Images. IEEE Access 7:114862-114887. doi:10.1109/ACCESS.2019.2935912

Isambert H, Venier P, Maggs AC, Fattoum A, Kassab R, Pantaloni D, Carlier M-F. 1995. Flexibility of Actin Filaments Derived from Thermal Fluctuations: EFFECT OF BOUND NUCLEOTIDE, PHALLOIDIN, AND MUSCLE REGULATORY PROTEINS*. Journal of Biological Chemistry 270:11437-11444. doi:10.1074/jbc.270.19.11437

Iwatsuki H, Suda M. 2010. Seven Kinds of Intermediate Filament Networks in the Cytoplasm of Polarized Cells: Structure and Function. Acta Histochemica Et Cytochemica 43:19-31. doi:10.1267/ahc. 10009

Jacob JT, Coulombe PA, Kwan R, Omary MB. 2018. Types I and II Keratin Intermediate Filaments. Cold Spring Harb Perspect Biol 10:a018275. doi:10.1101/cshperspect.a018275 
626

627

628

629

630

631

632

633

634

635

636

637

638

639

640

641

642

643

644

645

646

647

648

649

650

651

652

653

654

655

656

657

658

659

660

661

662

663

664

665

666

667

Karsch S, Büchau F, Magin TM, Janshoff A. 2020. An intact keratin network is crucial for mechanical integrity and barrier function in keratinocyte cell sheets. Cell Mol Life Sci 77:4397-4411. doi:10.1007/s00018-019-03424-7

Kim Y-B, Hlavaty D, Maycock J, Lechler T. 2021. Roles for Ndel1 in keratin organization and desmosome function. $M B o C$ mbc.E21-02-0087. doi:10.1091/mbc.E21-02-0087

Kothari P, Johnson C, Sandone C, Iglesias PA, Robinson DN. 2019. How the mechanobiome drives cell behavior, viewed through the lens of control theory. J Cell Sci 132:jcs234476. doi: $10.1242 /$ jcs. 234476

Kreplak L, Bär H, Leterrier JF, Herrmann H, Aebi U. 2005. Exploring the Mechanical Behavior of Single Intermediate Filaments. Journal of Molecular Biology 354:569-577. doi:10.1016/j.jmb.2005.09.092

Laha B, Bowman DA, Schiffbauer JD. 2013. Validation of the MR Simulation Approach for Evaluating the Effects of Immersion on Visual Analysis of Volume Data. IEEE Transactions on Visualization and Computer Graphics 19:529-538. doi:10.1109/TVCG.2013.43

Latorre E, Kale S, Casares L, Gómez-González M, Uroz M, Valon L, Nair RV, Garreta E, Montserrat N, del Campo A, Ladoux B, Arroyo M, Trepat X. 2018. Active superelasticity in threedimensional epithelia of controlled shape. Nature 563:203-208. doi:10.1038/s41586-0180671-4

Lee C-H, Kim M-S, Chung BM, Leahy DJ, Coulombe PA. 2012. Structural basis for heteromeric assembly and perinuclear organization of keratin filaments. Nat Struct Mol Biol 19:10.1038/nsmb.2330. doi:10.1038/nsmb.2330

Leube RE, Moch M, Windoffer R. 2017. Intracellular Motility of Intermediate Filaments. Cold Spring Harb Perspect Biol 9:a021980. doi:10.1101/cshperspect.a021980

Li H, Shen T, Smith MB, Fujiwara I, Vavylonis D, Huang X. 2009a. Automated actin filament segmentation, tracking and tip elongation measurements based on open active contour models2009 IEEE International Symposium on Biomedical Imaging: From Nano to Macro. Presented at the 2009 IEEE International Symposium on Biomedical Imaging: From Nano to Macro. pp. 1302-1305. doi:10.1109/ISBI.2009.5193303

Li H, Shen T, Vavylonis D, Huang X. 2010. Actin Filament Segmentation Using Spatiotemporal Active-Surface and Active-Contour Models In: Jiang T, Navab N, Pluim JPW, Viergever MA, editors. Medical Image Computing and Computer-Assisted Intervention - MICCAI 2010, Lecture Notes in Computer Science. Berlin, Heidelberg: Springer. pp. 86-94. doi:10.1007/978-3-642-15705-9_11

Li H, Shen T, Vavylonis D, Huang X. 2009b. Actin Filament Tracking Based on Particle Filters and Stretching Open Active Contour Models In: Yang G-Z, Hawkes D, Rueckert D, Noble A, Taylor C, editors. Medical Image Computing and Computer-Assisted Intervention - MICCAI 2009, Lecture Notes in Computer Science. Berlin, Heidelberg: Springer. pp. 673-681. doi:10.1007/978-3-642-04271-3_82

Liberali P, Pelkmans L. 2012. Towards quantitative cell biology. Nat Cell Biol 14:1233-1233. doi:10.1038/ncb2648

Lidke DS. 2017. Quantitative cell biology: uniting disciplines to understand the cell. Mol Biol Cell 28:3133. doi:10.1091/mbc.E17-09-0551 
668

669

670

671

672

673

674

675

676

677

678

679

680

681

682

683

684

685

686

687

688

689

690

691

692

693

694

695

696

697

698

699

700

701

702

703

704

705

706

707

708

Lippincott-Schwartz J. 2014. Quantitative cell biology: transforming the conceptual, theoretical, instrumental, and methodological approaches to cell biology. Mol Biol Cell 25:3437. doi:10.1091/mbc.E14-08-1297

Liu Y, Kolagunda A, Treible W, Nedo A, Caplan J, Kambhamettu C. 2019. Intersection to Overpass: Instance Segmentation on Filamentous Structures With an Orientation-Aware Neural Network and Terminus Pairing Algorithm2019 IEEE/CVF Conference on Computer Vision and Pattern Recognition Workshops (CVPRW). Presented at the 2019 IEEE/CVF Conference on Computer Vision and Pattern Recognition Workshops (CVPRW). pp. 125-133. doi:10.1109/CVPRW.2019.00021

Lutz A, Jung D, Diem K, Fauler M, Port F, Gottschalk K, Felder E. 2020. Acute effects of cell stretch on keratin filaments in A549 lung cells. The FASEB Journal 34:11227-11242. doi:https://doi.org/10.1096/fj.201903160RR

Magliaro C, Callara AL, Vanello N, Ahluwalia A. 2019. Gotta Trace 'em All: A Mini-Review on Tools and Procedures for Segmenting Single Neurons Toward Deciphering the Structural Connectome. Front Bioeng Biotechnol 0. doi:10.3389/fbioe.2019.00202

Martin I, Moch M, Neckernuss T, Paschke S, Herrmann H, Marti O. 2016. Both monovalent cations and plectin are potent modulators of mechanical properties of keratin K8/K18 networks. Soft Matter 12:6964-6974. doi:10.1039/C6SM00977H

Moccia S, De Momi E, El Hadji S, Mattos LS. 2018. Blood vessel segmentation algorithms Review of methods, datasets and evaluation metrics. Computer Methods and Programs in Biomedicine 158:71-91. doi:10.1016/j.cmpb.2018.02.001

Moch M, Herberich G, Aach T, Leube RE, Windoffer R. 2013. Measuring the regulation of keratin filament network dynamics. Proc Natl Acad Sci U S A 110:10664-10669. doi:10.1073/pnas. 1306020110

Moch M, Schwarz N, Windoffer R, Leube RE. 2020. The keratin-desmosome scaffold: pivotal role of desmosomes for keratin network morphogenesis. Cell Mol Life Sci 77:543-558. doi:10.1007/s00018-019-03198-y

Moch M, Windoffer R, Schwarz N, Pohl R, Omenzetter A, Schnakenberg U, Herb F, Chaisaowong K, Merhof D, Ramms L, Fabris G, Hoffmann B, Merkel R, Leube RE. 2016. Effects of Plectin Depletion on Keratin Network Dynamics and Organization. PLoS One 11:e149106. doi:10.1371/journal.pone.0149106

Nafeey S, Martin I, Felder T, Walther P, Felder E. 2016. Branching of keratin intermediate filaments. Journal of Structural Biology 194:415-422. doi:10.1016/j.jsb.2016.03.023

Nowak J, Gennermann K, Persson S, Nikoloski Z. 2020. CytoSeg 2.0: automated extraction of actin filaments. Bioinformatics 36:2950-2951. doi:10.1093/bioinformatics/btaa035

Osmanagic-Myers S, Gregor M, Walko G, Burgstaller G, Reipert S, Wiche G. 2006. Plectincontrolled keratin cytoarchitecture affects MAP kinases involved in cellular stress response and migration. J Cell Biol 174:557-568. doi:10.1083/jcb.200605172

Özdemir B, Reski R. 2021. Automated and semi-automated enhancement, segmentation and tracing of cytoskeletal networks in microscopic images: A review. Computational and Structural Biotechnology Journal 19:2106-2120. doi:10.1016/j.csbj.2021.04.019 
709

710

711

712

713

714

715

716

717

718

719

720

721

722

723

724

725

726

727

728

729

730

731

732

733

734

735

736

737

738

739

740

741

742

743

744

745

746

747

748

749

750

Parajón E, Surcel A, Robinson DN. 2021. The mechanobiome: a goldmine for cancer therapeutics. American Journal of Physiology-Cell Physiology 320:C306-C323. doi:10.1152/ajpcell.00409.2020

Piston DW. 2014. Biophysical Journal Special Issue: Focus on Quantitative Cell Biology. Biophys J 107:E01. doi:10.1016/j.bpj.2014.10.038

Portet S. 2013. Dynamics of in vitro intermediate filament length distributions. Journal of Theoretical Biology 332:20-29. doi:10.1016/j.jtbi.2013.04.004

Portet S, Leduc C, Etienne-Manneville S, Dallon J. 2019. Deciphering the transport of elastic filaments by antagonistic motor proteins. Phys Rev E 99:042414. doi:10.1103/PhysRevE.99.042414

Portet S, Madzvamuse A, Chung A, Leube RE, Windoffer R. 2015. Keratin Dynamics: Modeling the Interplay between Turnover and Transport. PLoS One 10:e0121090. doi:10.1371/journal.pone.0121090

Quan T, Zhou H, Li J, Li S, Li A, Li Y, Lv X, Luo Q, Gong H, Zeng S. 2016. NeuroGPS-Tree: automatic reconstruction of large-scale neuronal populations with dense neurites. Nature Methods 13:51-54. doi:10.1038/nmeth.3662

Quinlan RA, Schwarz N, Windoffer R, Richardson C, Hawkins T, Broussard JA, Green KJ, Leube RE. 2017. A rim-and-spoke hypothesis to explain the biomechanical roles for cytoplasmic intermediate filament networks. J Cell Sci 130:3437-3445. doi:10.1242/jcs.202168

Roca-Cusachs P, Conte V, Trepat X. 2017. Quantifying forces in cell biology. Nat Cell Biol 19:742751. doi:10.1038/ncb3564

Samuel PM, Veeramalai T. 2020. Review on retinal blood vessel segmentation - an algorithmic perspective. International Journal of Biomedical Engineering and Technology 34:75-105. doi:10.1504/IJBET.2020.110362

Schwarz N, Windoffer R, Magin TM, Leube RE. 2015. Dissection of keratin network formation, turnover and reorganization in living murine embryos. Scientific Reports 5:9007. doi:10.1038/srep09007

Sternberg S. 2012. Curvature in Mathematics and Physics, 1st ed. Dover Publications, Inc.

Strnad P, Stumptner C, Zatloukal K, Denk H. 2008. Intermediate filament cytoskeleton of the liver in health and disease. Histochem Cell Biol 129:735-749. doi:10.1007/s00418-008-0431-X

Takatsuki H, Bengtsson E, Månsson A. 2014. Persistence length of fascin-cross-linked actin filament bundles in solution and the in vitro motility assay. Biochimica et Biophysica Acta (BBA) General Subjects 1840:1933-1942. doi:10.1016/j.bbagen.2014.01.012

Tateishi K, Nishida T, Inoue K, Tsukita S. 2017. Three-dimensional Organization of Layered Apical Cytoskeletal Networks Associated with Mouse Airway Tissue Development. Sci Rep 7:43783. doi:10.1038/srep43783

te Molder L, Kreft M, Heemskerk N, Schuring J, de Pereda JM, Wilhelmsen K, Sonnenberg A. 2021. EGFR-dependent tyrosine phosphorylation of integrin $\beta 4$ is not required for downstream signaling events in cancer cell lines. Sci Rep 11:8675. doi:10.1038/s41598-021-88134-6

Wagner OI, Rammensee S, Korde N, Wen Q, Leterrier J-F, Janmey PA. 2007. Softness, strength and self-repair in intermediate filament networks. Exp Cell Res 313:2228-2235. doi:10.1016/j.yexcr.2007.04.025 
751

752

753

754

755

756

757

758

759

760

761

762

763

764

765

766

767

768

769

770

771

772

773

774

775

776

777

778

779

780

781

782

783

784

785

786

787

788

Wang J, Lü D, Mao D, Long M. 2014. Mechanomics: an emerging field between biology and biomechanics. Protein Cell 5:518-531. doi:10.1007/s13238-014-0057-9

Weber MS, Eibauer M, Sivagurunathan S, Magin TM, Goldman RD, Medalia O. 2021. Structural heterogeneity of cellular K5/K14 filaments as revealed by cryo-electron microscopy. eLife 10:e70307. doi:10.7554/eLife.70307

Wöll S, Windoffer R, Leube RE. 2005. Dissection of keratin dynamics: different contributions of the actin and microtubule systems. European Journal of Cell Biology 84:311-328. doi:10.1016/j.ejcb.2004.12.004

Xu T, Langouras C, Koudehi MA, Vos BE, Wang N, Koenderink GH, Huang X, Vavylonis D. 2019. Automated Tracking of Biopolymer Growth and Network Deformation with TSOAX. Scientific Reports 9:1717. doi:10.1038/s41598-018-37182-6

Xu T, Li H, Shen T, Ojkic N, Vavylonis D, Huang X. 2011. Extraction and analysis of actin networks based on Open Active Contour models2011 IEEE International Symposium on Biomedical Imaging: From Nano to Macro. Presented at the 2011 IEEE International Symposium on Biomedical Imaging: From Nano to Macro. pp. 1334-1340. doi:10.1109/ISBI.2011.5872647

Xu T, Vavylonis D, Tsai F-C, Koenderink GH, Nie W, Yusuf E, Lee I-J, Wu J-Q, Huang X. 2015. SOAX: A software for quantification of 3D biopolymer networks. Scientific Reports 5:9081. doi:10.1038/srep09081

Yamada S, Wirtz D, Coulombe PA. 2003. The mechanical properties of simple epithelial keratins 8 and 18: discriminating between interfacial and bulk elasticities. J Struct Biol 143:45-55. doi:10.1016/s1047-8477(03)00101-1

\section{Conflict of Interest}

The authors declare that the research was conducted in the absence of any commercial or financial relationships that could be construed as a potential conflict of interest.

\section{ETHICS STATEMENT}

n.a.

\section{Author Contributions}

- Conceptualization: RW, JDR, REL

- Methodology: RW

- Software: RW, MS, JS, AB

- Validation: RW

- Formal analysis: RW

- Investigation: RW, NS, SY, TP

- Resources: RW 


\section{$9 \quad$ Funding}

796 The work was supported by the German Research Council (LE566/18-2; WI173/8-2;

797 GRK2415/363055819).

798

799

800

801

802

803

804

805

806

807

808

809

810

811

812

813

814

815

816

817

818

819

820

821

822

823

824

825

826

827

828

- Writing - original draft: RW, REL

- Writing — review \& editing: RW, REL

- Visualization: RW, AB

- Supervision: RW

- Project administration: RW, REL

- Funding acquisition: RW, REL

Calculations were performed with computing resources granted by RWTH Aachen University under project rwth0452.

J.D.R and T.P. were supported by a grant from the Interdisciplinary Centre for Clinical Research within the faculty of Medicine at the RWTH Aachen University.

\section{Acknowledgments}

We gratefully acknowledge the helpful discussions and advice of Drs. Michael Schaub (RWTH Aachen University), Charlotte Lorenz and Sarah Köster (Göttingen University), Rudolf Merkel (FZ Jülich) and Michael Anhuth and Till Petersen-Krauß (RWTH Aachen University) for the implementation of the VR application.

\subsection{Figure Legends}

FIGURE 1 Evaluation of 3D keratin filament network segmentation. The pictures show three different numerical representations of the same fluorescence data set detecting the keratin filament network in an MDCK cell expressing fluorescently labelled keratin 8. The cell is part of a confluent monolayer and the fluorescence of the single cell shown was manually excised from an image stack consisting of 32 slices (voxel size: $x y=66 \mathrm{~nm}, \mathrm{z}=182 \mathrm{~nm}$ ). This cropped fluorescence was used to calculate the vertex positions and intensity of all keratin filaments. (A) The top picture shows an overlay of the recorded fluorescence in red as a maximum intensity projection (mip) and the corresponding calculated xy positions of vertices of all segments as green dots. The enlargements below (corresponding regions demarcated by boxes) show maximum intensity projections of focal planes above and below the nucleus (apical and basal) at left and two regions of interest (roi 1, roi 2) depicting maximum intensity projections of all focal planes in the cell periphery. (B) presents an overlay of the keratin fluorescence and corresponding vertices that were plotted in 3D with a diameter relative to their brightness and were subsequently subjected to 3D Gaussian blurring to simulate the microscope's blur. The enlargements correspond to those shown at left in (A) presenting the same regions of interest and focal planes. The top panel of Video 1 presents an animated comparison of the fluorescence recording, segmentation and overlay in each focal plane. (C) depicts a cinematic rendering of segments. Each calculated segment is rendered in 3D with a thickness corresponding to the brightness of the original filament. The color-coding helps to identify and distinguish individual segments. The regions of interest correspond to the same single planes as those shown in (A). White arrows demarcate thin filaments, blue arrows thick filaments. 
FIGURE 2 Comparison of 3D cinematic rendering of keratin filaments in three different epithelial cell types. The position and intensity of fluorescence recordings were transferred into a numerical 3D-representation of individual segments. The thickness of the segments corresponds to the fluorescence brightness in the original data. (A) shows cinematic rendering of keratin filaments of an MDCK cell expressing fluorescently labelled keratin 8. Video 2 presents the 3D-animation of the cell. (B) Depicts the cinematic rendering of keratin filaments in a HaCaT keratinocyte expressing fluorescently labelled keratin 5 (animation in Video 3). (C) Illustrates cinematic rendering of keratin filaments in a single RPE cell of a homozygous keratin 8-YFP knock-in mouse (animation in Video 4).

838 FIGURE 3 Visual inspection of the keratin network of a single cell in a virtual reality environment. The viewer can move freely within the network which appears to her/him as a true 3D stereoscopic structure. Classification of segments is done interactively in this environment by color coding.

841 FIGURE 4 Mapping of keratin filament brightness in MDCK, HaCaT and RPE cells. (A-C) The histograms display the distribution of relative segment brightness for each cell type. The maximum brightness was normalized to 100 in all cases. For each segment, the mean brightness was calculated and the determined brightness levels of all segments in 10 cells were pooled for each diagram. (D-F) The maps depict the segment brightness in a representative MDCK (left), HaCaT (middle) and RPE (right) cell (top panel; enlarged boxed area in bottom panel). The colour code represents relative brightness.

FIGURE 5 Measurements of keratin filaments in MDCK, HaCaT and RPE cells. (A-D) The whisker box plots depict the number of segments per cell (A), the mean segment length (B), the entire length of the keratin filament network within a single cell $(\mathbf{C})$ and the filament length per $\mu \mathrm{m}^{3}(\mathbf{D}) .10$ cells were analyzed for each cell type. (E-G) show color-coded keratin filament segment lengths for each cell type. The top panel presents the results for entire cells, the bottom panel the results for the boxed areas at higher magnification.

855 FIGURE 6 Calculation of keratin filament node types based on segment/node ratios in MDCK, $856 \mathrm{HaCaT}$ and RPE cells. A segment/node ratio of 3 refers to Y-shaped branching, a ratio of 4 to X857 shaped branchings. Higher values refer to star-like branchings. The histograms depict the relative 858 distribution of node types.

FIGURE 7 Measurements of keratin filament segment bending in MDCK, HaCaT and RPE cells.

860 (A). The whisker box plot shows the distance/length ratio of keratin segments. (B) The curvature of keratin filaments in the three cell types is shown as a whisker box plot. (C-E) Color-coded

862 representation of the keratin network depicting the curvature of keratin filament segments in entire cells (top) and selected regions of interest (boxed areas) at higher magnification (bottom).

864 FIGURE 8 Orientation of keratin filament segments in MDCK, HaCaT, and RPE cells. (A-C) The histograms show the normalized distribution of azimuth (= horizontal orientation) of keratin filament segments in single cells. Since the segments have no defined start and end point, the histogram is restricted to 180 degrees. The horizontal line represents the expected histogram levels if all angles were equally distributed. (D-F) The histograms show the elevation (= vertical orientation) of keratin filament segments. The horizontal line demarcates the expected histogram values, if elevation of keratin filament segments would be uniform. $(\mathbf{G}, \mathbf{H})$ Whisker box plot depicting the deviation of segment orientations from uniform unbiased distributions. The differences in azimuth $(\mathbf{G})$ and elevation (H) were calculated for 10 MDCK, $10 \mathrm{HaCaT}$ and $10 \mathrm{RPE}$ cells. (I-L) The color-coded 
873 maps depict the azimuth of segments in entire cells (top; MDCK, left; HaCaT, middle; RPE, right)

874 and selected regions of interest (boxed areas; bottom).

875 FIGURE 9 Analysis of segment orientation in MDCK, HaCaT and RPE cells. (A-F) The blue

876 polygons represent radial histograms of the orientation of keratin filament segments in relation to the 877 cell center of single cells. Horizontal orientation (A-C) and the vertical orientation (D-F) are shown.

878 Each keratin segment in each cell was moved to the cell center and vectors pointing in the same

879 directions were added. Thus, the longer the blue lines are, the more vectors point in that direction.

880 (G-I) The red arrows represent the sum vectors of the direction and length of all segments in each

881 cell. (K) Whisker box plots depicting the length of the main vectors in all three cell types.

882

883

884

FIGURE 10 Schematic drawing of the reconstructed keratin network in the three cell types

885

886

887 


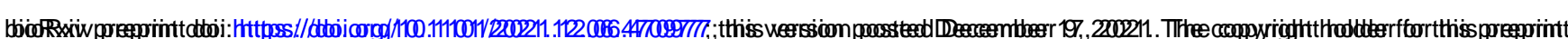

(which was not certified by peer review) is the author/funder, who has granted bioRxiv a license to display the preprint in perpetuity. It is made available under aCC-BY 4.0 International licen39-Mapping of Keratin Networks

88911 Figures

890

891

\subsection{Figure 01}
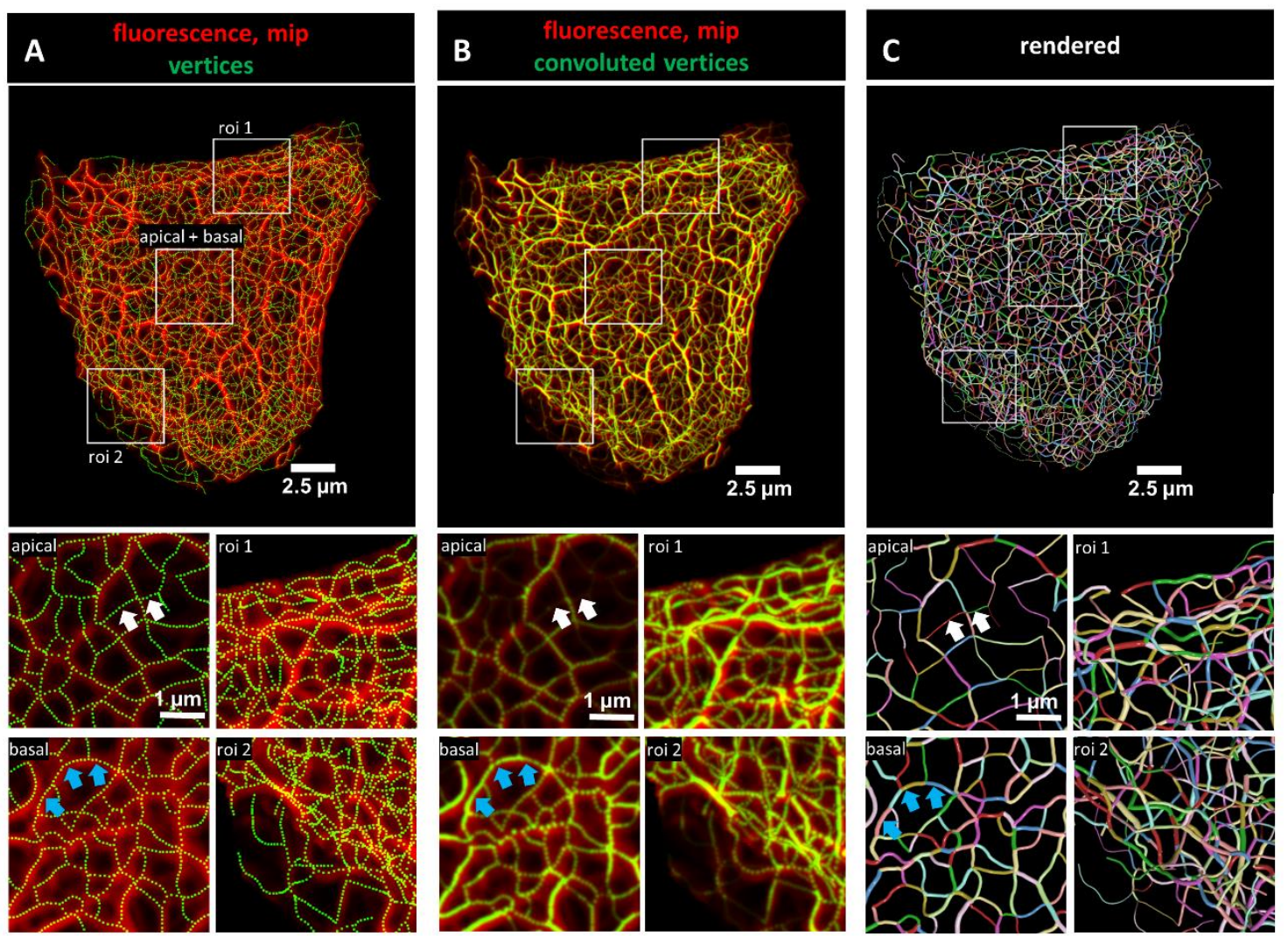

892

$893 \quad 11.2$ Figure 02 


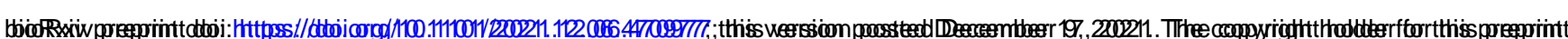
(which was not certified by peer review) is the author/funder, who has granted bioRxiv a license to display the preprint in perpetuity. It is made available under aCC-BY 4.0 International licen39-Mapping of Keratin Networks

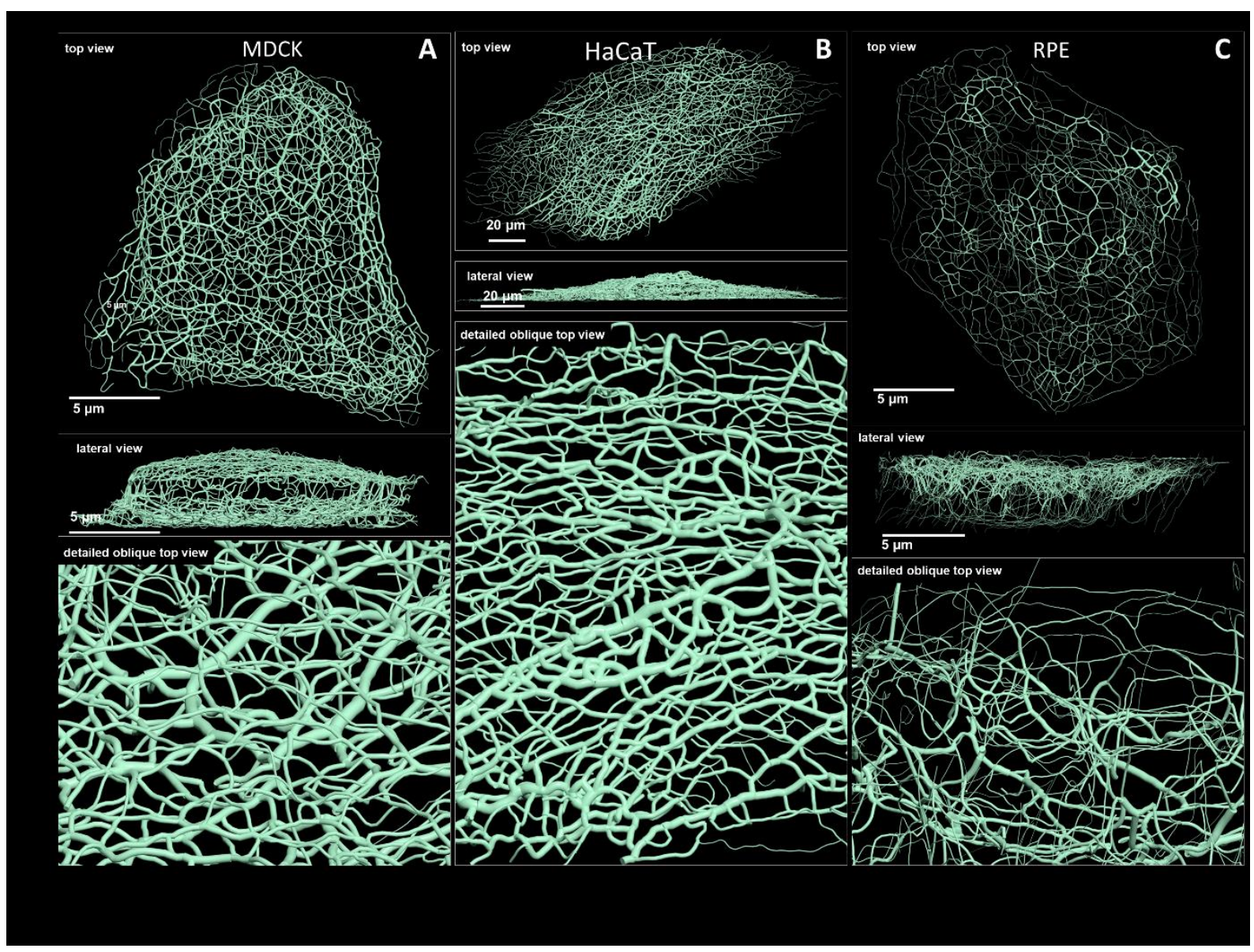

895

896

897 


\section{D-Mapping of Keratin Networks}

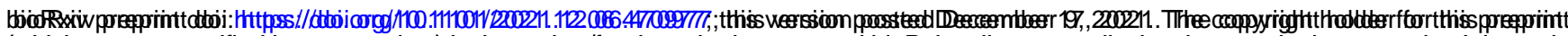
898 (which yas nptgertifiediby peer review) is the author/funder, who has granted bioRxiv a license to display the preprint in perpetuity. It is made 899

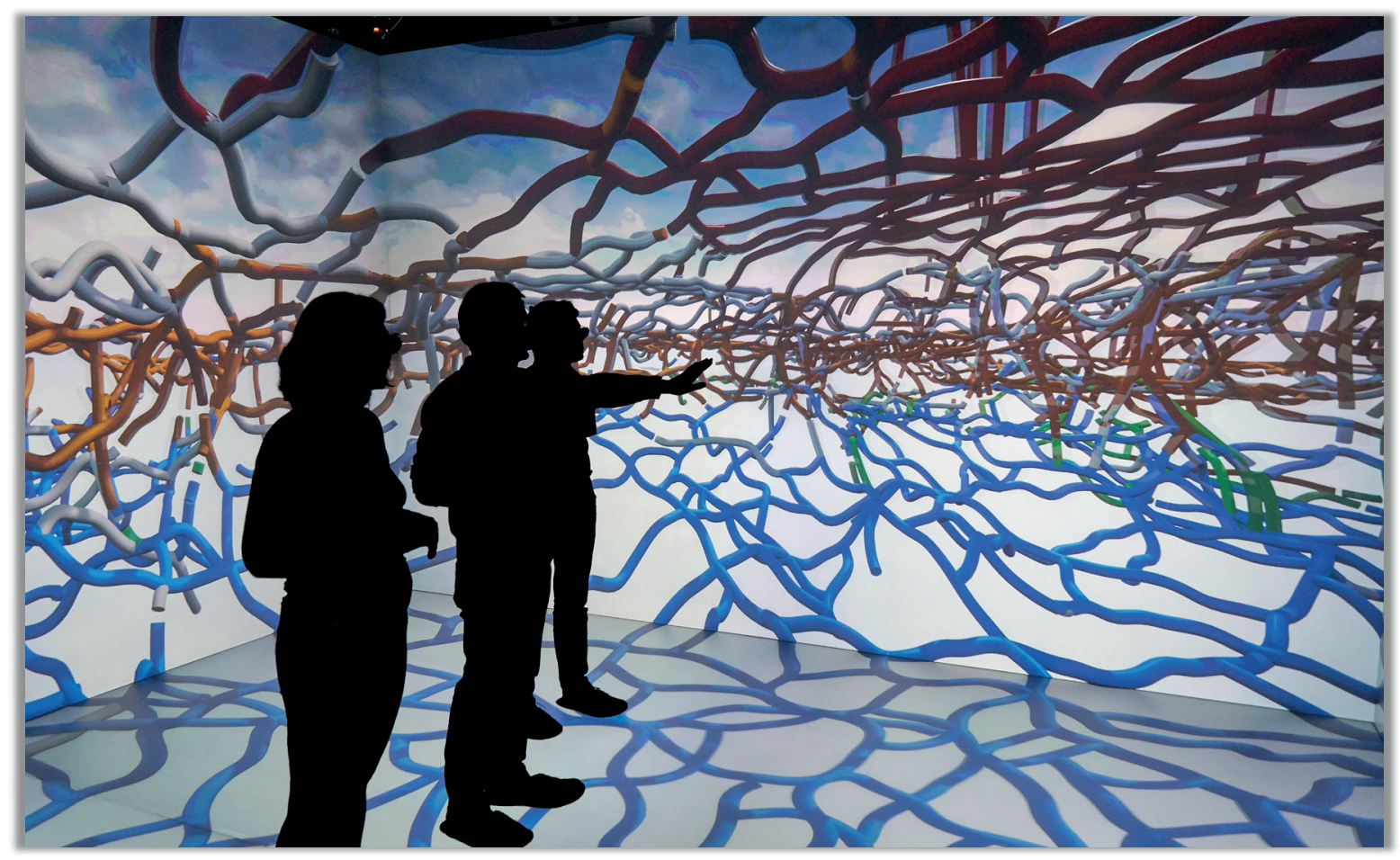




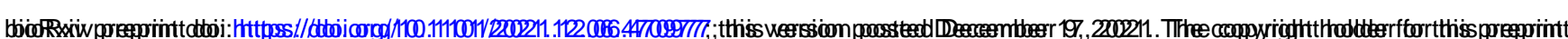

(which was not certified by peer review) is the author/funder, who has granted bioRxiv a license to display the preprint in perpetuity. It is made available under aCC-BY 4.0 International licen39-Mapping of Keratin Networks

903

\section{$904 \quad 11.4$ Figure 04}

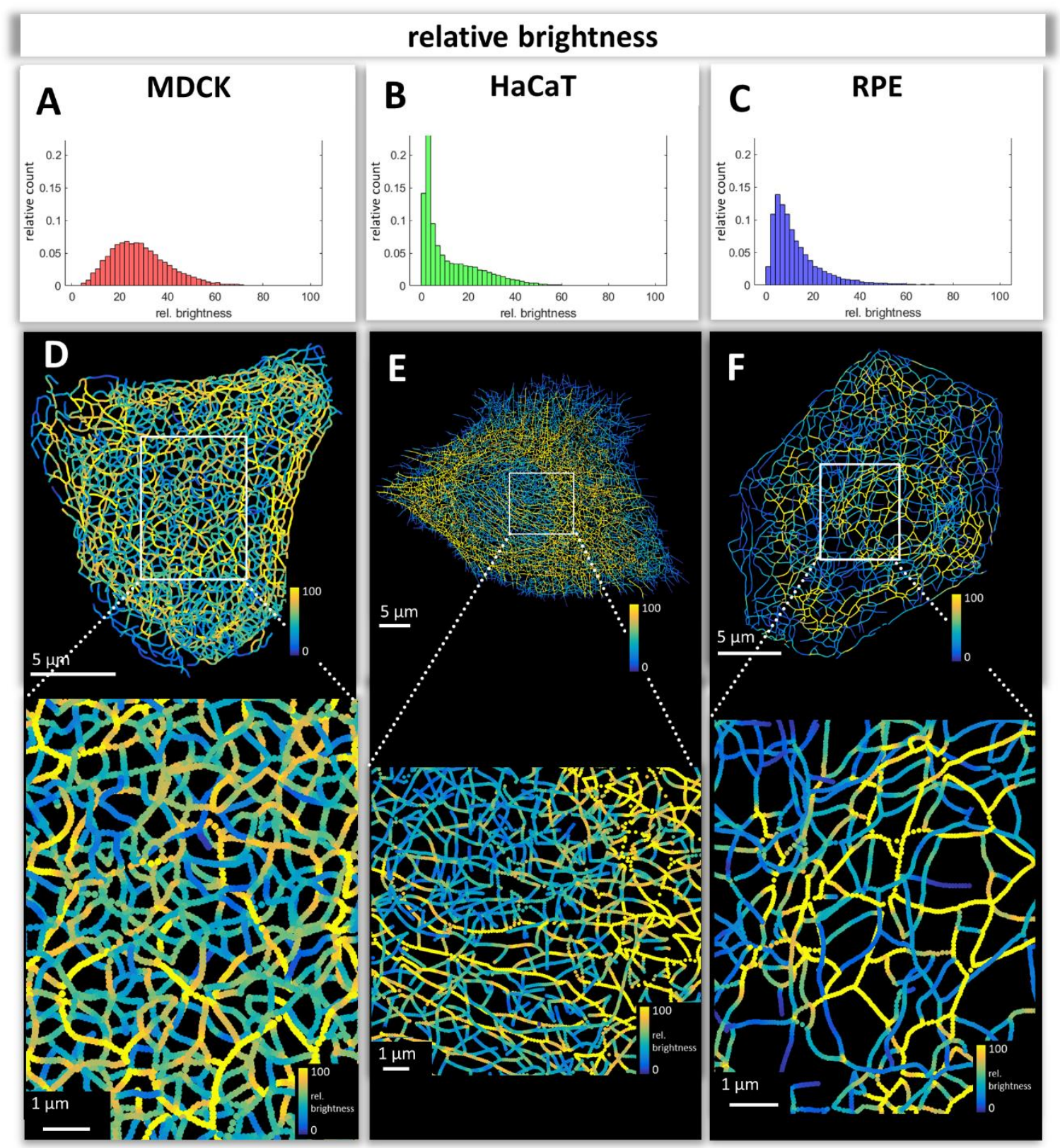

905

906 


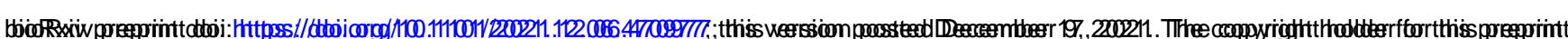
(which was not certified by peer review) is the author/funder, who has granted bioRxiv a license to display the preprint in perpetuity. It is made available under aCC-BY 4.0 International licen39-Mapping of Keratin Networks

\subsection{Figure 05}

908
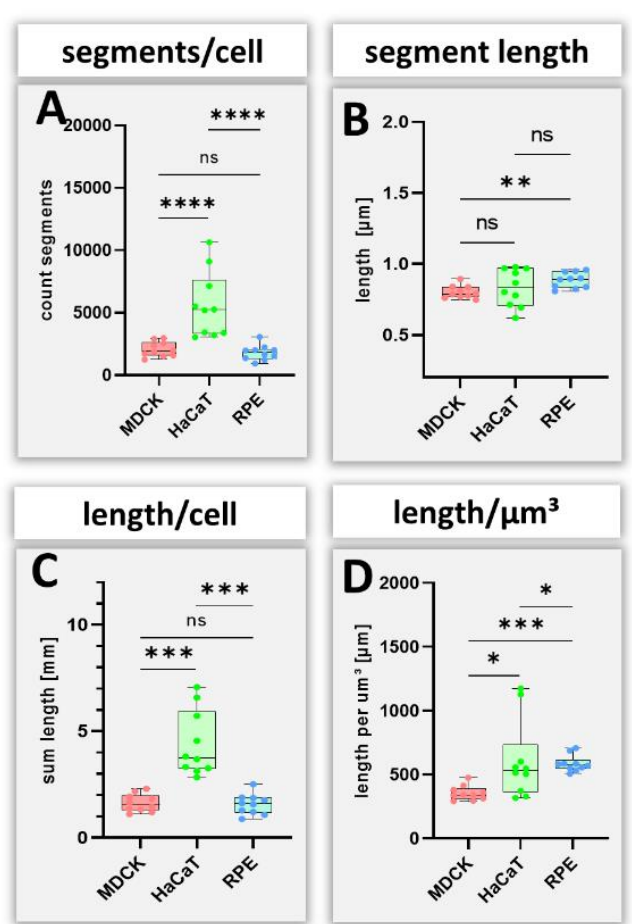

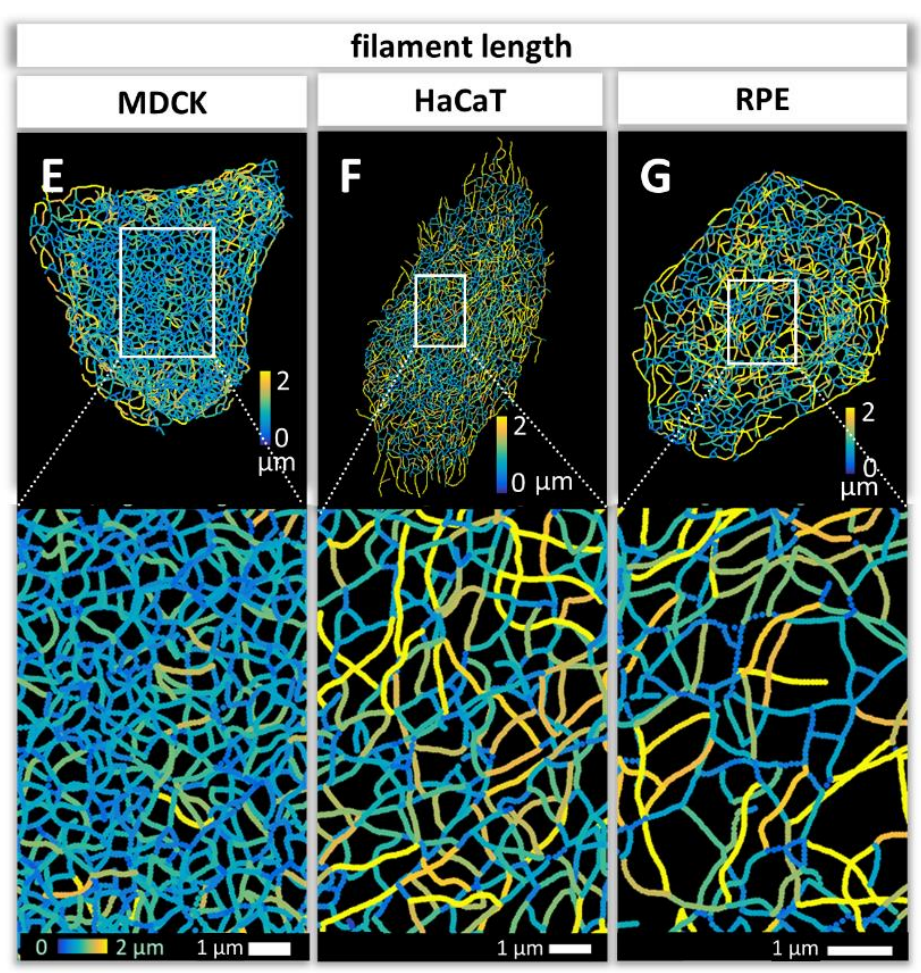

909

910

911 


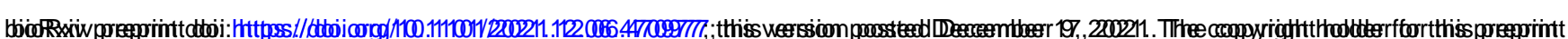
(which was not certified by peer review) is the author/funder, who has granted bioRxiv a license to display the preprint in perpetuity. It is made available under aCC-BY 4.0 International licen39-Mapping of Keratin Networks

912

\section{$913 \quad 11.6$ Figure 06}
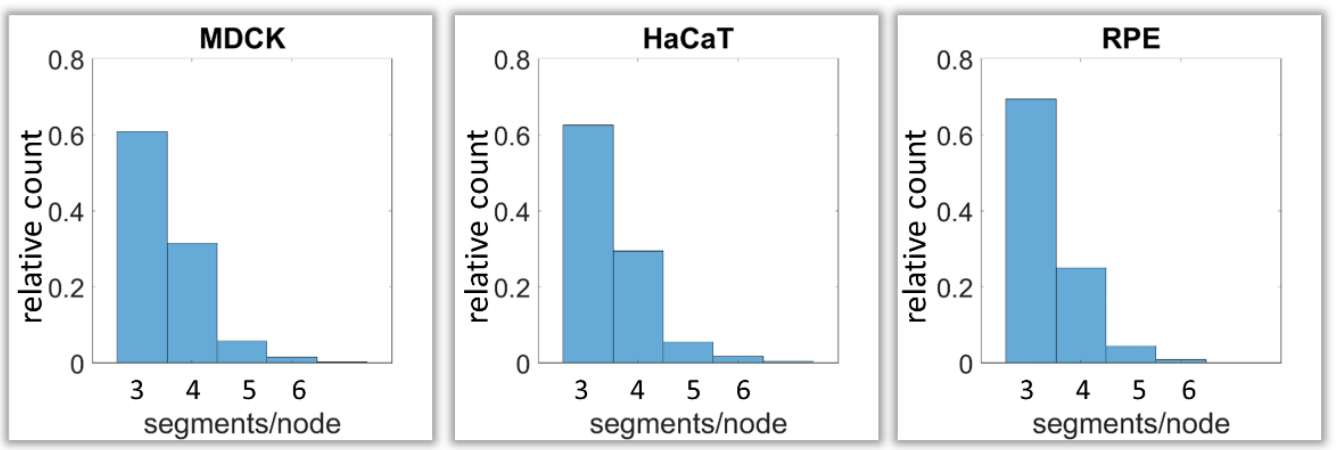

914

915 


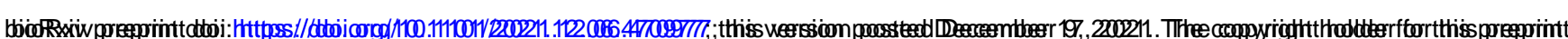

(which was not certified by peer review) is the author/funder, who has granted bioRxiv a license to display the preprint in perpetuity. It is made available under aCC-BY 4.0 International licen3D-Mapping of Keratin Networks
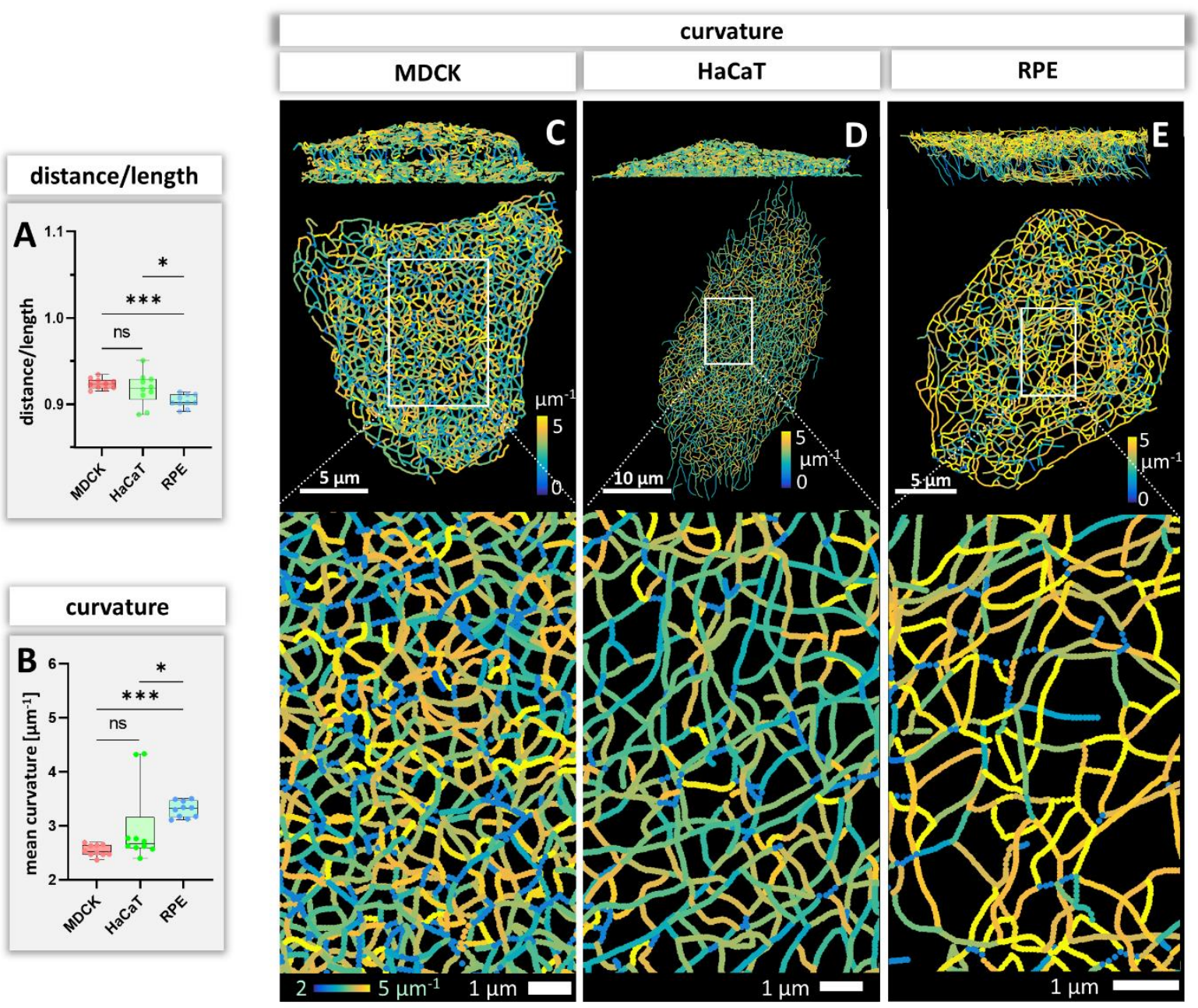

917

918

919 


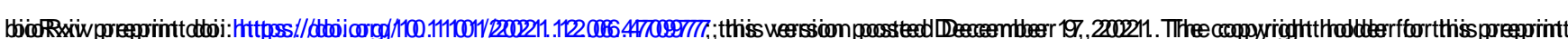
(which was not certified by peer review) is the author/funder, who has granted bioRxiv a license to display the preprint in perpetuity. It is made available under aCC-BY 4.0 International licen39-Mapping of Keratin Networks

\section{$921 \quad 11.8$ Figure 08}
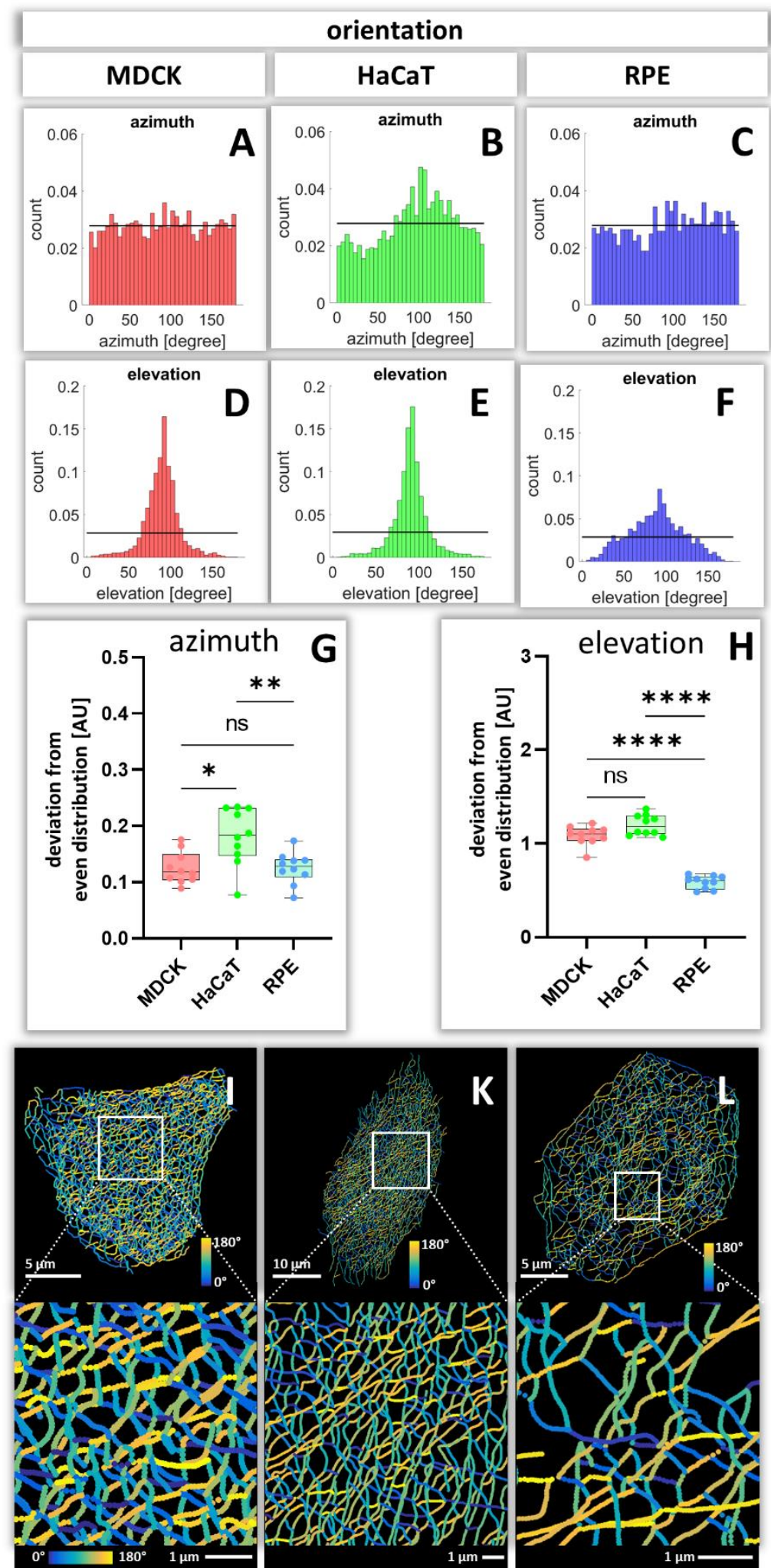


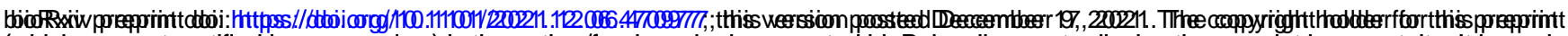
(which was not certified by peer review) is the author/funder, who has granted bioRxiv a license to display the preprint in perpetuity. It is made available under aCC-BY 4.0 International licen39-Mapping of Keratin Networks

$924 \quad 11.9$ Figure 09

segment orientation
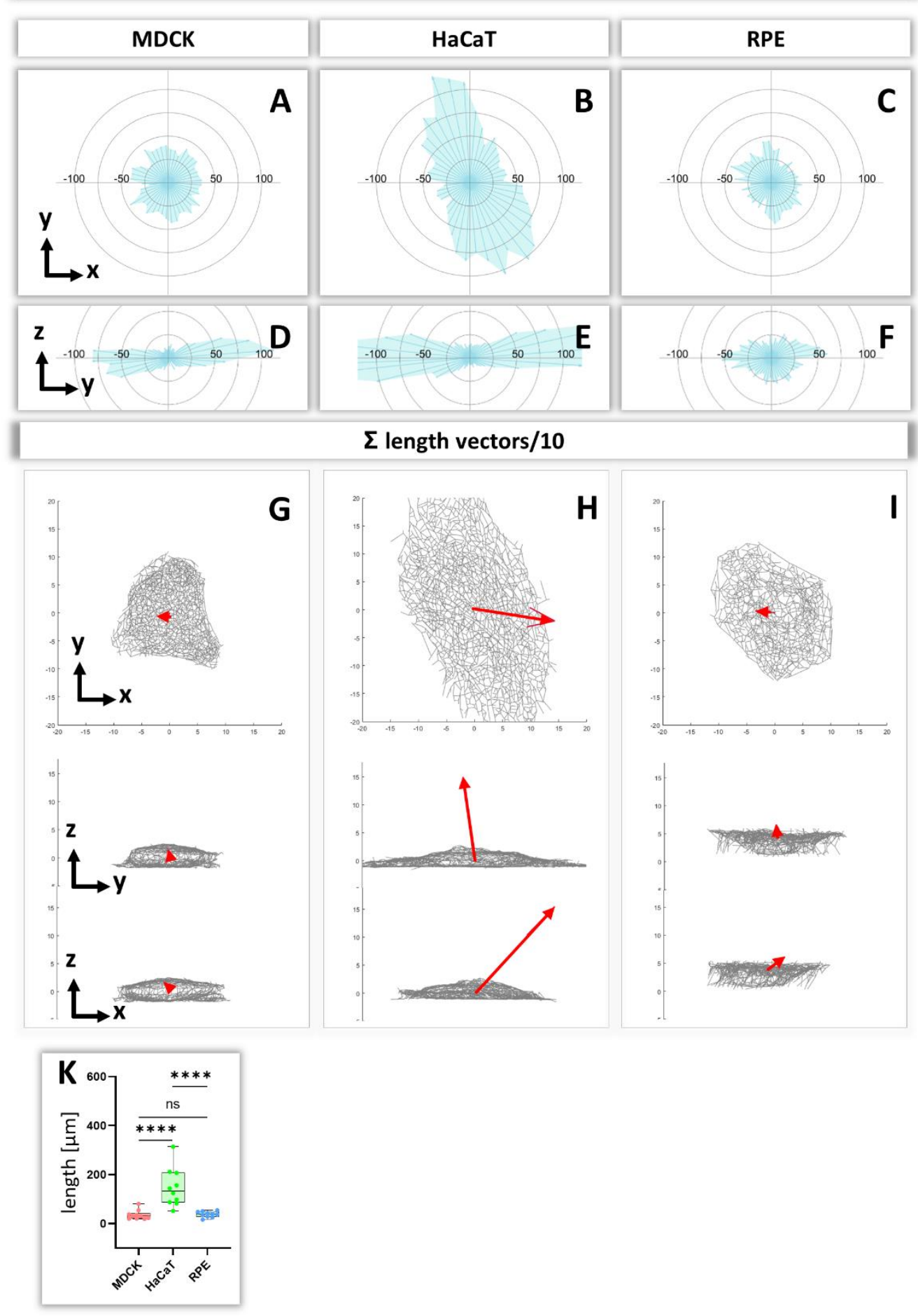

925
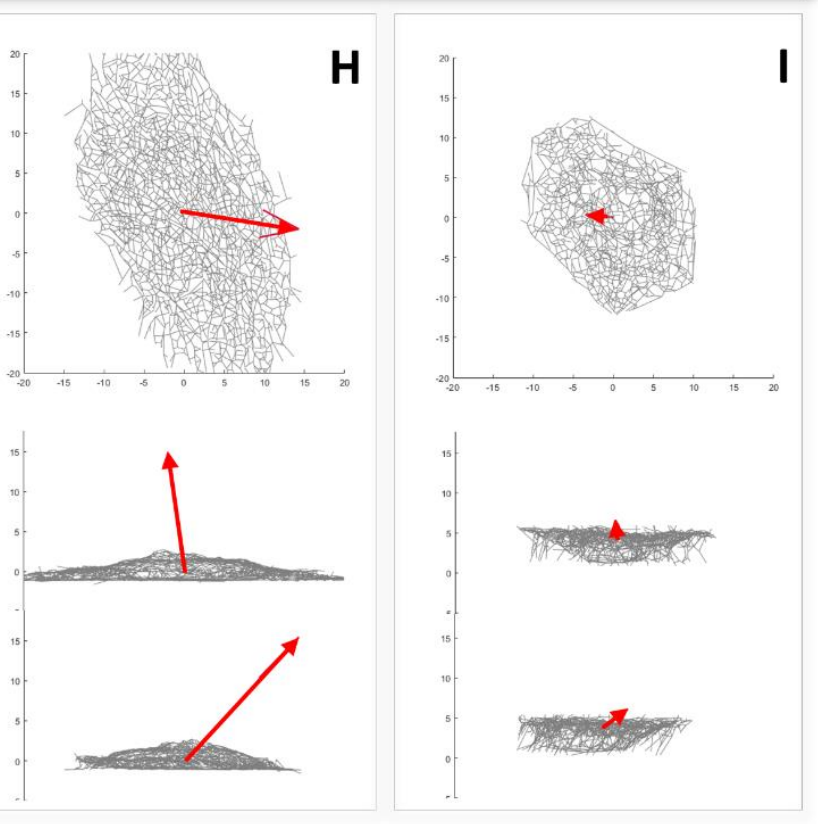

926

31 


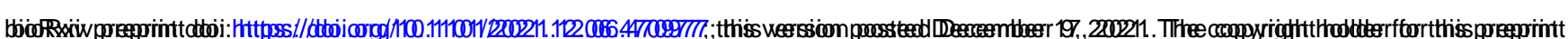
(which was not certified by peer review) is the author/funder, who has granted bioRxiv a license to display the preprint in perpetuity. It is made available under aCC-BY 4.0 International licen39-Mapping of Keratin Networks

\section{11.10 Figure 10}
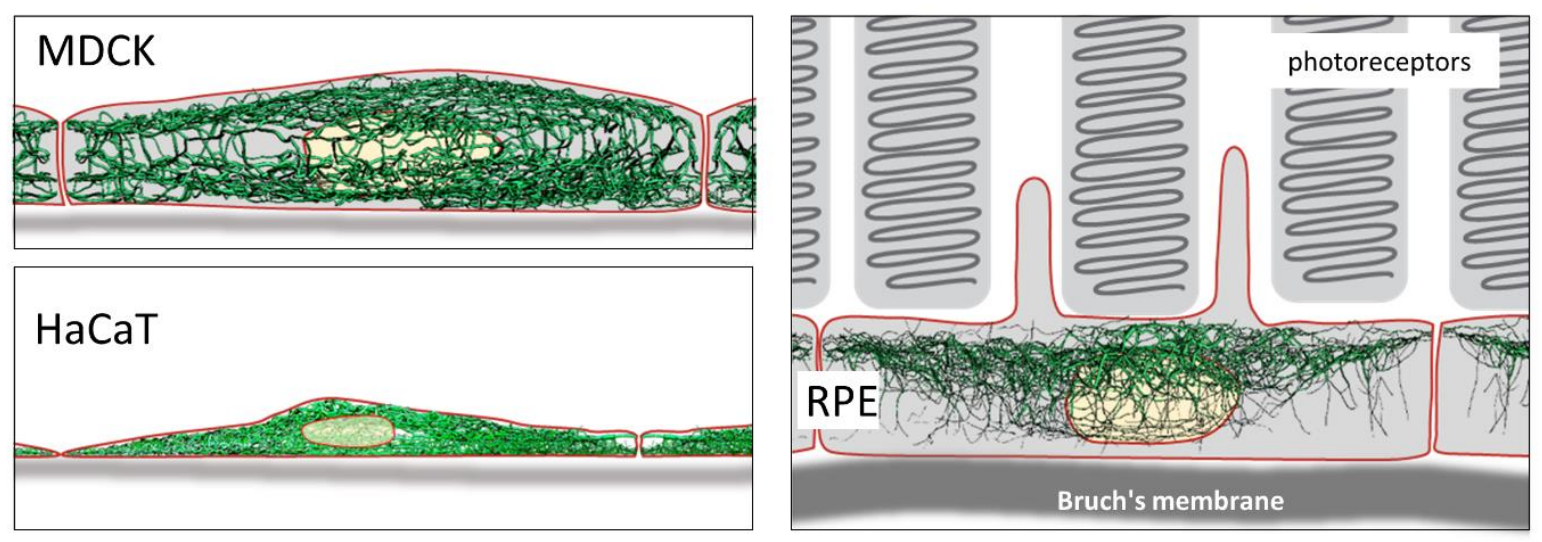


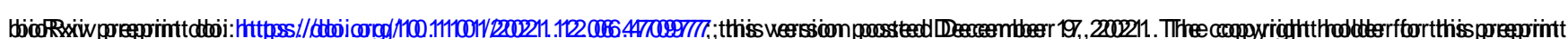
(which was not certified by peer review) is the author/funder, who has granted bioRxiv a license to display the preprint in perpetuity. It is made available under aCC-BY 4.0 International licen3థ-Mapping of Keratin Networks 